\title{
Polymer Viscosity, Particle Coalescence and Mechanical Performance in High Temperature Laser Sintering
}

\author{
S. Berretta ${ }^{1}$, Y. Wang ${ }^{1}$, R. Davies ${ }^{1}$ and O.R. Ghita ${ }^{1}$ \\ ${ }^{1}$ University of Exeter, College of Engineering, M athematics and Physical Science, Exeter EX4 4QF, UK
}

\begin{abstract}
High Temperature Laser Sintering (HT-LS) is an additive manufacturing technology whose potential could be limited by the restricted number of materials optimised for the process. Poly Ether Ether Ketone (PEEK) with different melt viscosity values, PEEK 150PF and PEEK 450PF, have been used in parallel with the commercial grade, Poly Ether Ketone (PEK) HP3, to investigate the role of material viscosity on particle coalescence, parts structure and mechanical performance in HT-LS. The material with lower viscosity, PEEK 150PF, was found to exhibit faster coalescence and lower tensile strength than the grades with higher viscosities, PEEK 450PF and PEK HP3.
\end{abstract}

\section{Keywords}

PEEK, PEK, High Temperature Laser Sintering, viscosity, crystallinity, tensile, storage, particle coalescence

\section{Introduction}

Within the range of additive manufacturing technologies, Laser Sintering (LS) is one of the most mature and advanced fabrication methods being used primarily with various grades of polyamides (PA) and few other engineering polymers [1, 2]. Recently, High Temperature Laser Sintering (HT-LS) emerged as a process able to fabricate complex Poly Ether Ether Ketone (PEEK) and Poly Ether Ketone (PEK) components. Part of the Poly Aryl Ether Ketone (PAEK) family, PEEK and PEK polymers are known to compete in performance with metals, being known for their exceptional properties: resistant to corrosion, abrasion, stress-fatigue and ionising radiation, low toxicity and toughness.

Due to their unique characteristics, PAEK polymers lend themselves as good materials to ducting and bracketry parts, engine and under-bonnet components, piston rings and gears, flexible displays and smart devices, gaskets and solid pipe solutions, radiation protection equipment and cranial and spinal cages implants.

Using the EOSINT P 800 system, LS of commercial HP3 PEK grade and off-the-shelf Victrex PEEK 150PF and 450PF powders has been proven [3-9], however these studies mostly focussed in assessing the mechanical performance and microstructure of the laser sintered parts rather than the particle coalescence or the polymer viscosity.

Beard et al. [3] examined the microstructure, thermal stability, surface roughness and mechanical performance of HT-LSPEK HP3 samples and found them to exhibit high thermal resistance and a tensile strength above $90 \mathrm{MPa}$.

Ghita et al. [8] studied virgin and used (material that has already been through the HT-LS manufacturing process) PEK HP3 particles in terms of particle sintering mechanism, melting temperature, crystallisation 
temperature and mechanical performance of the laser sintered components. HT-LS PEK HP3 samples made with used powder showed a tensile strength drop of 17\% between PEK HP3 pure virgin powder. Ghita et al. [7] also completed the analysis of PEK HP3 in EOSINT P 800 by investigating the mechanical, shrinkage and degradation properties of over 250 PEK HP3 test specimens manufactured along the $X, Y$ and $Z$ axes. Samples manufactured along the $X$ and $Y$ axes showed data in accordance with the results of Beard et al. [3]. However, a drop of nearly $50 \%$ in the tensile strength was found in the samples manufactured along the $Z$ axis. Wang et al. [9] analysed thermal and mechanical properties of laser sintered components made of PEK HP3 and glass bead fillers.

Berretta et al. [4] provided a systematic investigation into the HT-LS processing of PEEK within the EOSINT P 800. Laser sintered PEEK 45OPF samples manufactured with different processing parameters and in varied building configurations were analysed in terms of microstructure, surface roughness, thermal and mechanical performance. PEEK samples showed a $25 \%$ lower tensile strength than laser sintered PEK samples but same storage modulus.

The relationship between material properties, processing parameters and parts properties is at the heart of a good manufacturing process. As with many manufacturing processes, the tendency is to focus on optimising process parameters and parts performance rather than to define starting material properties and their behaviour within the process.

In the case of the LS process, a powder with a good flow and appropriate particle size distribution leads to good powder spreading performance and particle packing, this subsequently leads to more dense parts and less porosity [10].

Choosing the right polymer viscosity is equally important, as a polymer with good melt flow characteristics can lead to a fast and high neck growth and good particle coalescence. At the same time, polymer viscosity can promote layer to layer adhesion [1] and therefore influence the mechanical strength of the parts produced due to the molecular chain characteristics [11]. Selection of a low polymer viscosity leads to rapid particle sintering but creates parts of low mechanical performance and with higher occurrence of shrinkage in the geometry [2]. In contrast, a high molecular weight polymer, which has a high melt viscosity, leads to slow particle sintering and potentially reduced neck growth but in general fabricates parts of higher mechanical strength.

A limited number of studies investigated these interactions and defined these polymer characteristics for polyamides only [1, 2, 10, 12]. According to Kruth et al. [2] the ratio between surface tension and melt viscosity, velocity parameter, defines the limit for a successful sintering in polymers. Materials like PA 12 that have a melt viscosity of $100 \mathrm{~Pa} \cdot \mathrm{s}$ can be fully sintered under surface tension of $30 \mathrm{mN} / \mathrm{m}$ thanks to the obtained velocity equal of $3 \cdot 10^{-4} \mathrm{~m} / \mathrm{s}$, whereas polymers like Polycarbonate with melt viscosity of $5000 \mathrm{~Pa} \cdot \mathrm{s}$ and dissimilar surface tension cannot. Conversely, the same authors report that polymers with the highest molecular weight as possible (and therefore highest molecular viscosity as possible) will allow manufacture of "strong" components with highest dimensional accuracy and least shrinkage [2]. Indeed, Shi et al. [10] studied the effect of several material properties including viscosity for two grades of polystyrene (PS) with different melt molecular weights. The authors suggest that the molecular weight, which affects directly the melt viscosity of a molten material, disrupts also the quality of laser sintered components. The specimens built with the lower melting viscosity PS grade exhibited higher densities than the specimens built with the higher viscous grade.

A common approach in the LS community is also to recommend polymeric powders exhibiting a wide temperature operating window $[1,13]$, i.e. a temperature interval between the onset temperatures of melting and crystallisation. This window called "Super-cooling window" [13] is defined on the basis of the thermal properties of melting and crystallisation of the material under test in relation to the temperature at which the 
material is held during a LS building operation. If the LS processing temperature is lower than the material onset crystallisation temperature and therefore located outside the window, major and localised shrinkage will occur during and just after the laser exposure that, in turn, will lead to deleterious effects on the mechanical properties of the laser sintered parts. If the LS processing temperature is higher than the material onset melting temperature, therefore again outside this proposed window, uncontrolled melting will occur all across the powder bed without even the laser exposure $[2,13,14]$. Any LS processing temperature inside the window instead will lead to controlled and effective sintering without any undesired and detrimental thermal event in the powder. It is largely believed that it will be easier to optimise processing temperatures for LS a material having this wide temperature window.

The super cooling window has been thought to be critical for laser sintering of semi-crystalline polymers. However, this is not the case. Thermal profiles of PEEK and PEK showed that sintering is well possible in absence of such operating window. Similarly, laser sintering of polystyrene [15], an amorphous polymeric structure presenting only glass transition temperature at approximately $90^{\circ} \mathrm{C}$, has been successfully laser sintered. And also commercial elastomeric LS materials such as thermoplastic poly urethane TPU 92A-1 from Materialise [16] and DURAFORM Flex @grades [17] can still be processed in the LS process without showing a super cooling window.

However in order to develop new material and tailored existing grades for processability into the HT-LS, a better understanding on the role of particle morphology, thermal properties, polymer viscosity on particle sintering and mechanical performance of $\mathrm{HT}$ laser sintered components needs to be accomplished, hence the study proposed here.

\section{Experimental work}

\subsection{Materials}

The materials utilized for characterisation and specimen manufacturing have been divided in: powders for HT$\mathrm{LS}$ and pellet materials for Injection M oulding (IM).

\subsubsection{Powders materials for HT-LS}

High Temperature (HT) laser sintered samples were manufactured using three types of powdered materials: HP3 PEK (supplied by EOS [18]), PEEK 450PF and 150PF powders (both supplied by Victrex [19]). Both PEEK powders were thermally conditioned before use in the HT-LS system in order to improve the powder flow [20]. The main properties of the powders proposed for investigation, the chemical structure and the melt viscosities identified in the literature, are presented in Table 1. The two PEEK powder grades (150PF and 450PF) have different molecular weight [21] and therefore different melt viscosity values, while HP3 PEK and PEEK grades differ in melt viscosity and chemical structure.

\begin{tabular}{|c|l|l|l|}
\hline Powder & Chemical structure & $\begin{array}{l}\text { Melt } \\
\text { Viscosity } \\
\text { (Pa.s) }\end{array}$ \\
\hline $\begin{array}{c}\text { PEEK } \\
450 \mathrm{PF}\end{array}$ &
\end{tabular}




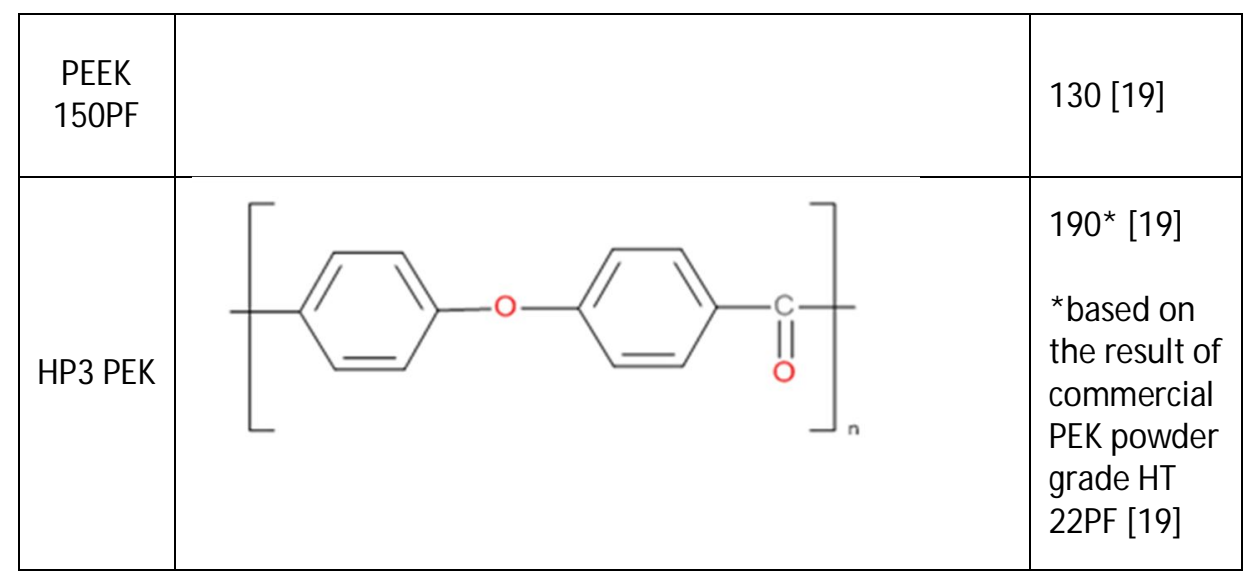

Table 1. M aterial properties of PEEK 450PF, PEEK 150PF and PEK HP3 powders

\subsubsection{Pellet material for IM}

The material used for the manufacture of IM samples was VICTREX HT G22, an established IM PEK grade. PEK pellets were dried for 5 hours at $120^{\circ} \mathrm{C}$ before use. The IM HT G22 PEK grade is equivalent to the HT-LS grade PEK HP3. According to the material's datasheet [19] this material has a melt viscosity of $200 \mathrm{~Pa} \cdot \mathrm{s}$.

\subsection{Sample manufacturing processes}

\subsubsection{Injection moulding (IM)}

Injection-moulded samples for tensile and Dynamic Mechanical Analysis (DM A) testing were manufactured as per manufacturer specifications using the HT G22 PEK grade and used as benchmark for HP3 PEK specimens. The nozzle temperature was $400{ }^{\circ} \mathrm{C}$, injection hydraulic pressure was 900 bars for 2 seconds, the holding hydraulic pressure was 900 bars; injection speed was $60 \mathrm{~cm}^{3} \times \mathrm{s} \mathrm{s}^{-1}$ and the cooling time was 15 seconds. Tensile samples were manufactured with ISO 527-2-1A geometries. Samples for DM A were manufactured with nominal dimension of $30 \mathrm{~mm} \times 7 \mathrm{~mm} \times 2 \mathrm{~mm}$ with rectangular geometries.

\subsubsection{High-Temperature Laser Sintering (HT-LS)}

Testing samples were manufactured within the HT-LS system EOSINT P 800 in reduced chamber building configuration. A detailed description of the processing parameters can be found in previous publications $[7,8]$. Tensile samples were manufactured with ISO 527-2-1A geometries along the $x$ direction of the referencing system within the building chamber of the EOSINT P 800 system. Rectangular samples with dimension of $30 \times 7$ $\times 2 \mathrm{~mm}$ were also manufactured as testing specimens for DM A.

\subsection{Experimental methods}

\subsubsection{Scanning Electron Microscopy (SEM)}

The SEM examination of powder materials and laser sintered units was performed with a Hitachi S- 3200N scanning electron microscope. The powders were thinly spread on metallic stubs and then sputter coated with $10 \mathrm{~nm}$ of gold in order to improve the electron conductivity and thus obtaining high resolution images. The electron secondary imaging was set with an accelerating voltage of $25 \mathrm{kV}$. 


\subsubsection{Angle of Repose (AOR)}

$A O R$ is a test which quantifies the angle of a cone of bulk material over a flat surface assuming that each material has its own specific angle of repose. The cone is formed by dropping the material through a funnel of standard dimensions and the angle considered is the inner one formed between the slant height and the horizontal plane. The smaller the angle of repose is, the higher the flowability is. The test was designed by following the ASTM C144 standard [35]. The interior of the glass funnel was covered with paper in such a way to fit perfectly the angle of the funnel and to facilitate the flow of powder; the powders were added in small amounts; the powder was supplied through the funnel until the cone of deposited material reached the tip of the nozzle. The test was repeated six times on each material.

\subsubsection{Differential Scanning Calorimetry (DSC)}

DSC allows studying a wide range of materials thermal properties. M elting crystallization temperature and crystallinity contents of powder samples were analysed using the Mettler Toledo DSC 821e/700. The powders were heated from room temperature up to well-above their melting temperature with heating and cooling rates of $10{ }^{\circ} \mathrm{C} \mathrm{x} \mathrm{min}{ }^{-1}$, respectively. Nitrogen was used as protective gas with a flow of $50 \mathrm{ml} \mathrm{x} \mathrm{min}^{-1}$. The enthalpy of fusion value of $130 \mathrm{~J} \mathrm{x}^{-1}$ for $100 \%$ crystalline PEK and PEEK was found in the literature [22] and used for the calculation of the crystallinity content of the PEEK and PEK samples under test.

\subsubsection{X-Ray Diffraction (XRD)}

The XRD measurements were performed with Bruker D8 Advance XRD with copper anode at room temperature. XRD data were collected in the angular range where $2 \theta=10-32^{\circ}$. The step size of $2 \theta$ was $0.01^{\circ}$. Aluminum Oxide $\left(\mathrm{Al}_{2} \mathrm{O}_{3}\right)$ was used as a standard sample to measure the instrumental line broadening and to calibrate the diffractometer. The crystal size $(\mathrm{L})$ of various samples was calculated from Equation 1.

$$
L=\frac{K \lambda}{F W(s) \cos \theta}
$$

Equation 1. Crystal size

$\lambda$ is the wavelength of copper anode $(0.154 \mathrm{~nm}), K$ is constant $(K=1)$ and FW $(\mathrm{s})$ is the specimen peak broadening at half the maximum intensity in radians.

\subsubsection{Melt Flow Index (MFI)}

Melt Flow Index is a measure of the mass of molten polymeric material flown through a die of standardised dimensions in a time lap of ten minutes. M Fl is related to the viscosity of the tested material: the lower the MFI number is, the higher the viscosity of the polymer. The test was carried out using the Melt Flow Tester 6941 CEAST according to the ISO standard 1133 and ASTM standard D 1238-04 with a material heating time of 5 minutes. The testing temperature is a material dependent parameter and was chosen approximately $20^{\circ} \mathrm{C}$ above the melting temperature for each material. The M FI test was chosen over other techniques aimed to measure polymeric rheology because of its simplicity, velocity and common utilisation in industry. The test was aimed to check the viscosity performance of the received materials. 


\subsubsection{Hot stage microscopy}

Particle sintering or particle coalescence is the process where two or more adjacent particles form necks and bond together because of a reduction of the free surface energies of the particle involved [23]. This energy reduction can be triggered by a high pressure or an elevated temperature [24]. The sintering phenomenon starts with the formation of a neck bonding between two particles, this neck increases until the two individual particles are no longer visible and only one unit is formed. A graphical explanation is provided in Figure 1.

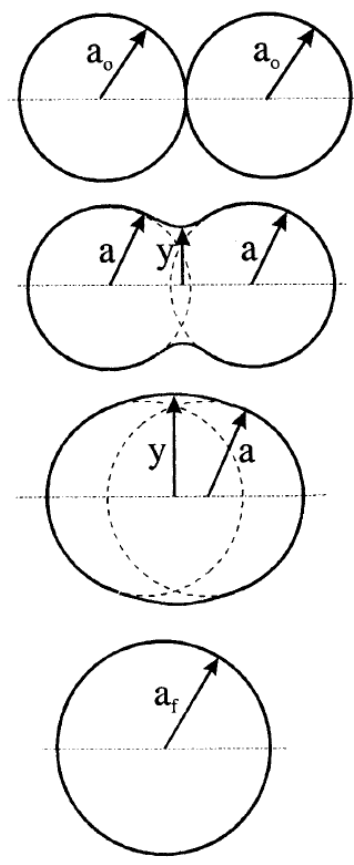

Figure 1. Particle sintering mechanism where $a_{0}$ initial particle radius, a particle radius, $a_{f}$ final particle radius and y neck radius [24]

The particle coalescence mechanism of the three powders PEK HP3, PEEK 150PF and PEEK 450PF was performed using the Linkam THM 5600 microscope stage placed under a Bruker IR Scope II, set in visual transmission mode and connected to a Dino Eye eyepiece camera Dino-Lite. The mechanism was studied for a two particle set-up for each material and experiment.

The aim of the hot stage microscopy tests was to study particle coalescence under experimental conditions that could simulate the HT-LS process, in order to compare sintering behaviour as a function of melt viscosity.

In the HT-LS process polymeric particles are consolidated by laser exposure followed by a layer heating phase called "Post Sintering" [4]. During a building operation, laser exposure and post sintering are recommended by the machine manufacturer EOS to account for an overall layer homogeneity which is likely to be connected to dimensional stability and accuracy of the components to be manufactured. The duration of laser exposure and post sintering time of a layer, also known as "Layer time", is suggested to be below $90 \mathrm{~s}$ (the term "Layer time" will be used by the authors when describing the coalescence process). The laser exposure is therefore responsible for increasing the material temperature above its melting point, causing its fusion (high final temperature) in a considerable short time (high heating rate). According to Vasquez [25], the heating rate of the $L S$ process of PA materials is roughly $22,000^{\circ} \mathrm{C} \times \mathrm{min}^{-1}$.

A few particles were spread on a microscope glass and inserted into the hot stage device. Two strategies were adopted in order to study neck formation of the materials under test. 
In the first approach, particles were heated from room temperature to $400{ }^{\circ} \mathrm{C}$ at $120^{\circ} \mathrm{Cx} \mathrm{min}{ }^{-1}$. This method allowed defining the onset temperature of the coalescence phenomenon under such heating rate.

In the second approach, the materials were heated from room temperature up to $400{ }^{\circ} \mathrm{C}$ at $120^{\circ} \mathrm{C} \mathrm{x} \mathrm{min}{ }^{-1}$ and held there for 2 minutes (120 s). In this way the particles under test were heated as fast as possible and exposed to high temperature for overall 2 minutes as best approximation of the recommended layer time in HT-LS processing. This method allowed simulating the building condition of every layer in the HT-LS process at best approximation.

Images of particle coalescence were taken approximately $20^{\circ} \mathrm{C}$ below the coalescence onset temperature previously found (using the first method) at 5 seconds interval for the PEK HP3 material and at 3 s time interval for the PEEK grades using Dino Capture 2.0 software. The different time intervals were chosen accordingly to the velocity at which each material was fully coalescing. M easurements of the neck formation were performed using the tool "Distance" in the Dino Capture software set at appropriate calibration and magnification parameters used in the tests. The first image presenting the formation of a necking bond was considered the beginning point of the coalescence. The end of coalescence phenomenon corresponded to no further changes in the neck formation and/or the addition of surrounding molten particles into the melt pool originated by the two particles under test. A minimum number of four repeats were performed for each material.

\subsubsection{Tensile testing}

Tensile samples were manufactured with ISO 527-2-1A geometries along the $x$ direction of the referencing system within the building chamber. Tensile testing experiments were performed by using a LLOYD instruments EZ20 mechanical testing machine at room temperature $\left(20^{\circ} \mathrm{C}\right)$. The testing speed was equal to 2

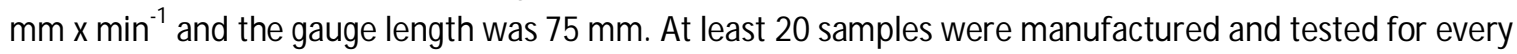
build. The average tensile strength and corresponding standard deviations were evaluated.

\subsubsection{Dynamic Mechanical Analysis (DMA)}

DM A allows the study of the viscoelastic behaviour of a given material by measuring the complex modulus. While the real part of the complex modulus represents the elastic behaviour (Storage modulus $E^{\prime}$ ) of a material, the imaginary part (Loss modulus, $E^{\prime \prime}$ ) represents its viscous performance. Tan $\delta$, defined as the ratio of loss modulus to storage modulus, represents the mechanical damping within a viscoelastic material. A lower $\tan \delta$ indicates a material that has high elastic strain component and vice versa. The tests were carried out using the Mettler Toledo DM A-1. All the specimens were built with nominal dimension of $30 \mathrm{~mm} \times 7 \mathrm{~mm} \times 2$ $\mathrm{mm}$ and tested in three points -bending mode (frequency $1 \mathrm{~Hz}$ and strain displacement $10 \mu \mathrm{m}$ ). During the test, each sample was heated from 30 to $300^{\circ} \mathrm{C}$ at heating rate of $3{ }^{\circ} \mathrm{C} \mathrm{x} \mathrm{min}{ }^{-1}$. At least, four repeats were carried out for each type of processing conditions.

\section{Experimental results}

\subsection{Particle morphology}

The SEM images of PEK HP3 particles are shown in Figure 2. 

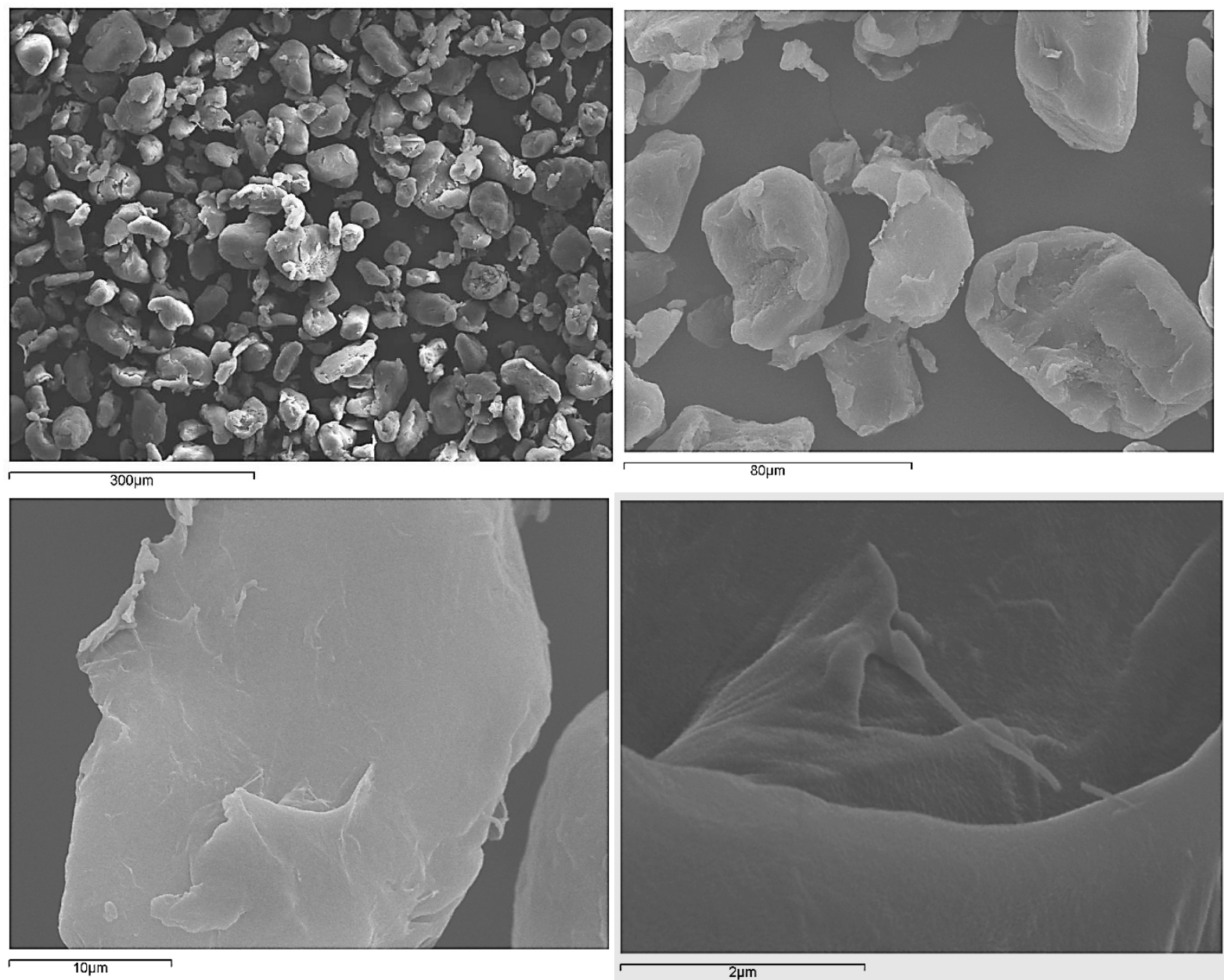

Figure 2. PEK HP3 particles at increasing order of magnification

PEK HP3 particles appear significantly round and circular with smooth surface and fully dense at increasing order of magnification.

Figure 3 and Figure 4 show the particles of thermally conditioned PEEK 450PF and PEEK 150PF, respectively. 


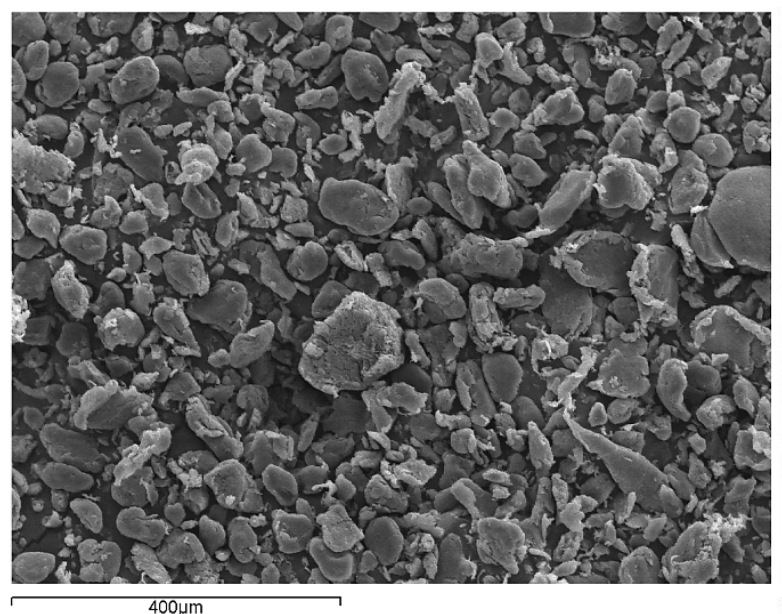

$400 \mu \mathrm{m}$

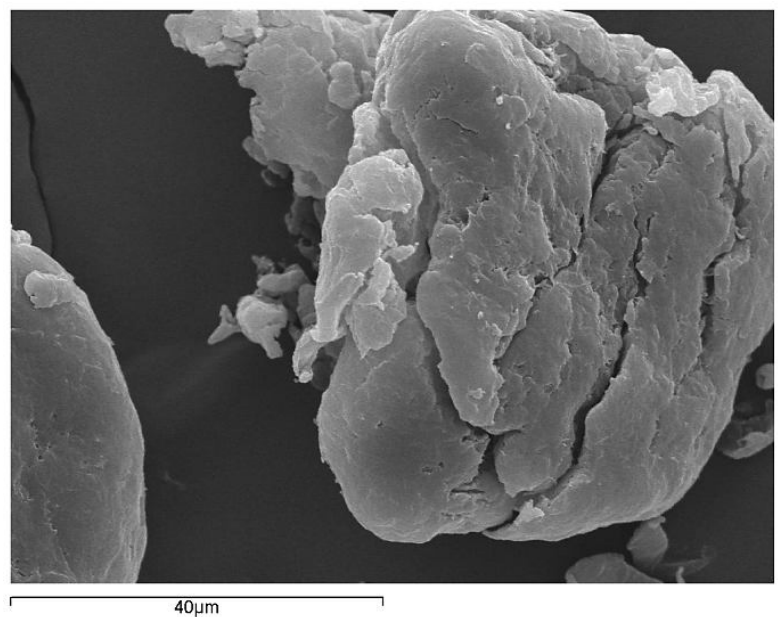

$40 \mu \mathrm{m}$

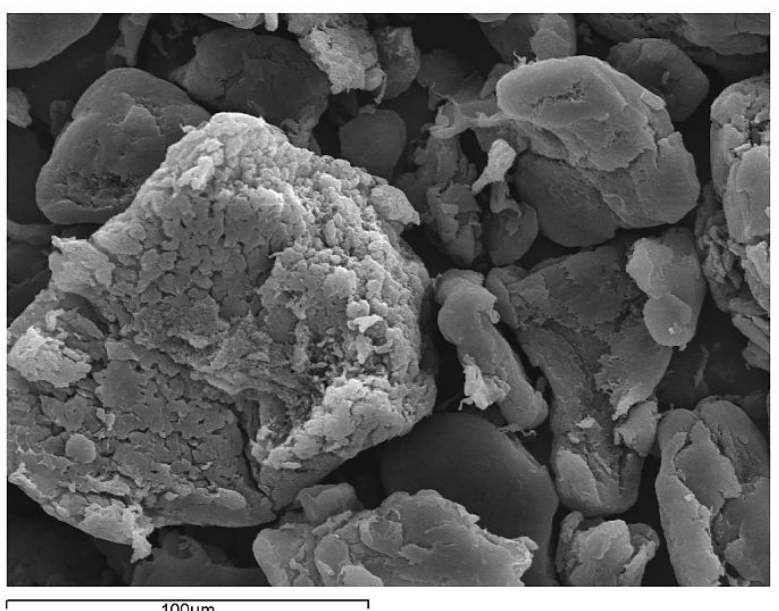

$100 \mu \mathrm{m}$

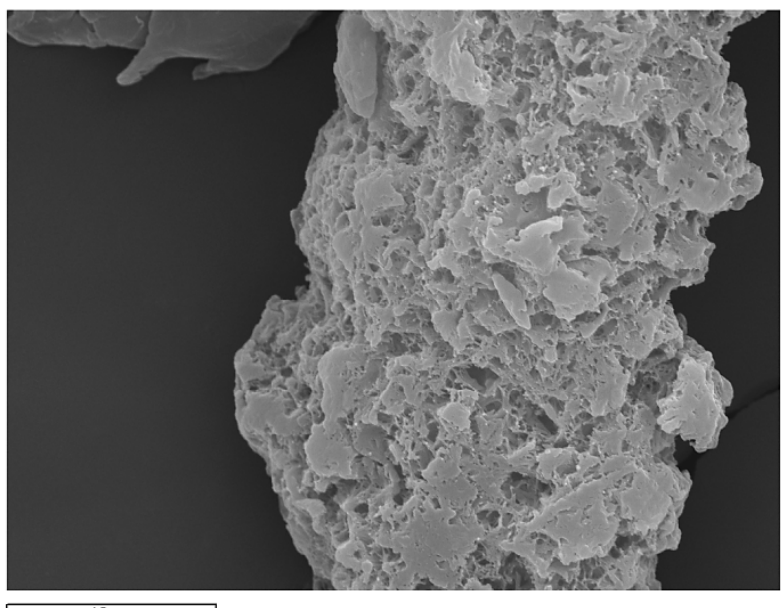

$10 \mu \mathrm{m}$

Figure 3. Thermally conditioned PEEK 450PF particles at increasing order of magnification 

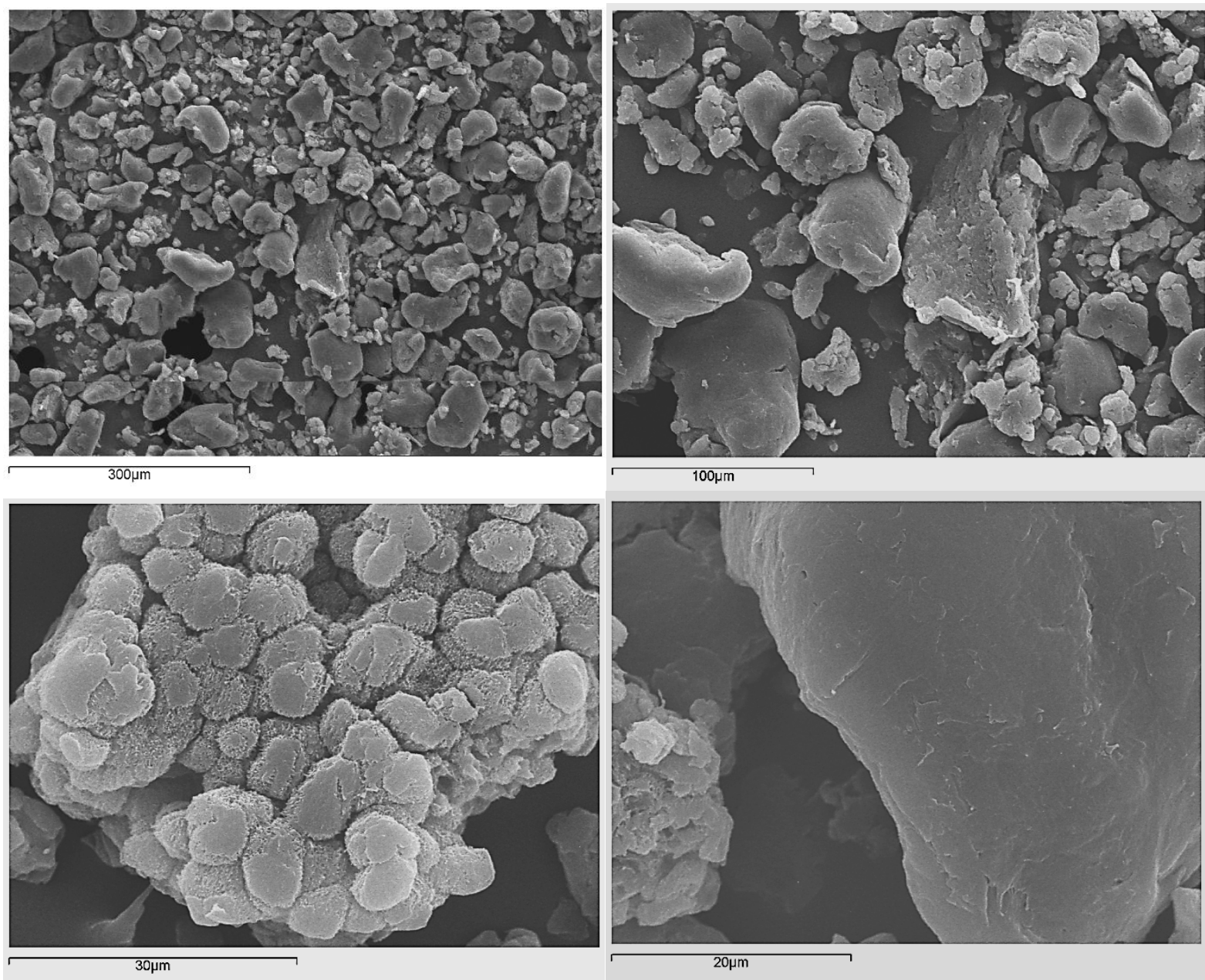

Figure 4. Thermally conditioned PEEK 150PF particles at increasing order of magnification

Both PEEK grades exhibit particles that are less round and circular when compared to the PEK HP3 material (Figure 2).

Berretta et al. [5] quantitatively analysed size and morphological characteristics of new potential candidate HT polymers and their composites in parallel with established Laser Sintering (LS) materials such as PEK HP3 and PA 2200. The study highlighted that a simple particle size analysis is not sufficient when searching for new HTLS powder candidates. A combined analysis of shape descriptors such as circularity, roundness, aspect ratio and solidity, inter-particle attractive forces and particle surface characteristics explained the differences found in the flow performance of the materials tested. Good flowability is one of the main and initial material requirements for good processability and components production in LS. Highly circular and round particles with smooth surfaces and fully dense structure were found to achieve higher flow behaviour. In a previous publication the authors showed that [5] particles with smoother surfaces, rounder and circular shapes exhibited a better flow behaviour. Circularity is a shape descriptor that quantifies how much a given particle shape is similar to a circle overall, roundness is instead a shape descriptor that indicates how round the edges of a particle are. PEEK particles present more complex structures and surfaces at increasing order of magnification then PEK HP3 particles. The centroids of the circularity and the roundness data distribution of more than a 1000 particles of PEEK 150PF, PEEK 450PF and PEH HP3 shown in Figure 5 has highlighted how PEEK 15OPF and PEK HP3 include rounder and more circular particles than PEEK 450PF. Furthermore, the thermal conditioning of the powder improves the particle morphology significantly, increasing the circularity and the roundness of the particles under treatment. The roundness and circularity data sets of PEEK 450PF 
before and after thermal conditioning is displayed in Figure 6 . The details of this quantitative analysis can be found elsewhere [5].

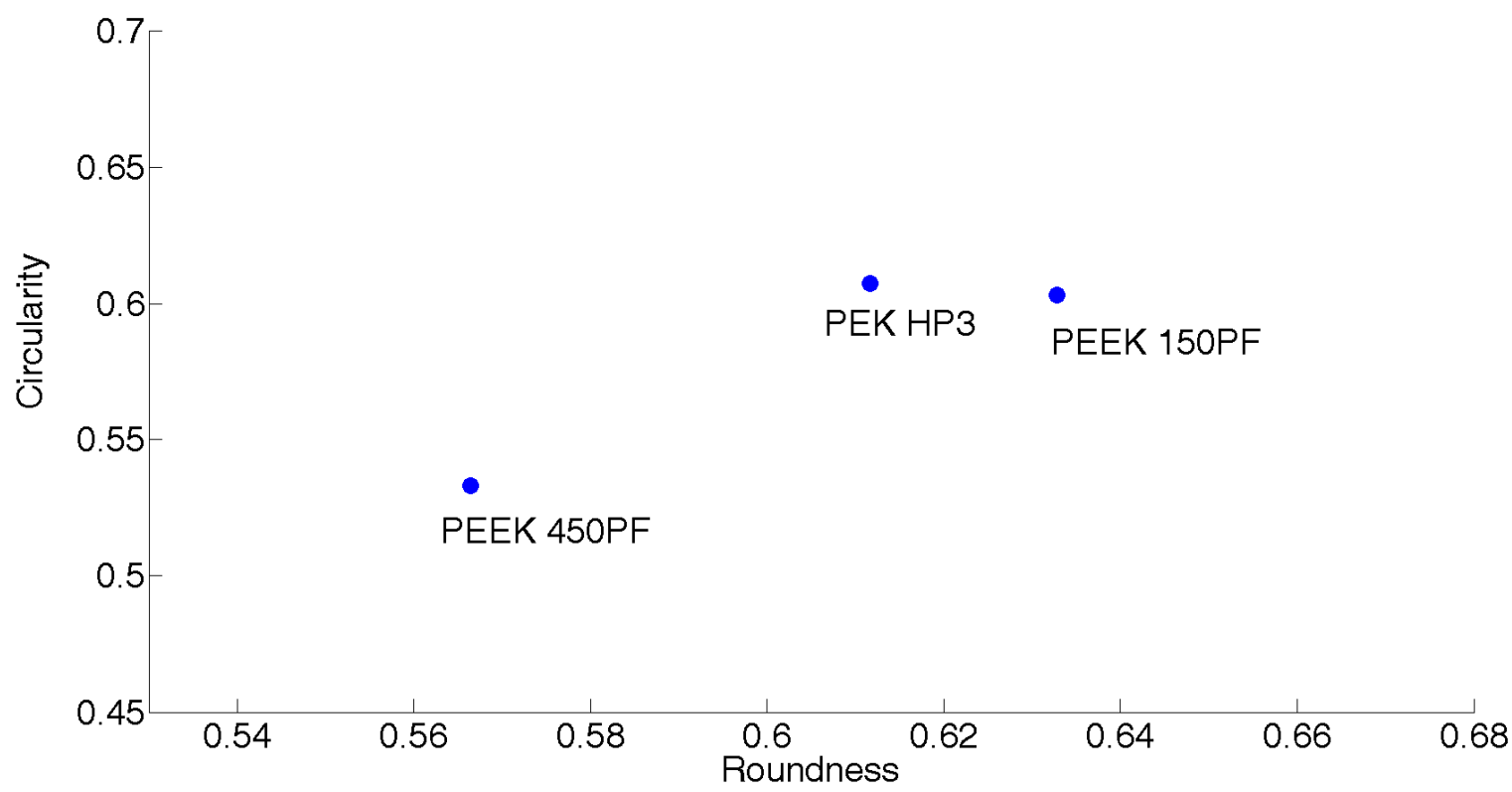

Figure 5. Centroids of the circularity and roundness data of PEEK 450PF, PEK HP3 and PEEK 150PF
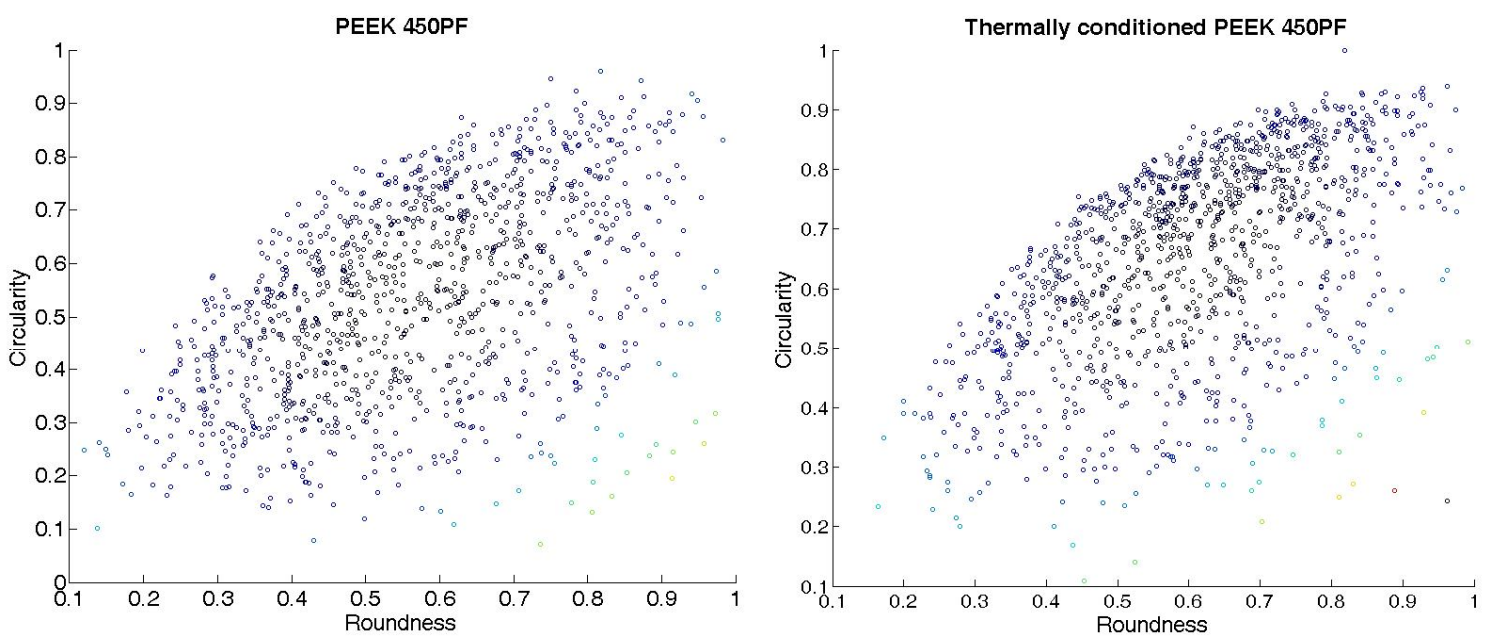

Figure 6. Change in the roundness and circularity data of PEEK 450PF and thermally conditioned PEEK 450PF

\subsection{AOR results}

The Angle of Repose (AOR) is a measurement of flowability of bulk materials. The results of the AOR test on PEK HP3, thermally conditioned PEEK 450PF and PEEK 150PF are listed in Table 2. 


\begin{tabular}{|l|l|}
\hline Material & AOR $\left(^{\circ}\right)$ \\
\hline PEK HP3 & $42 \pm 1$ \\
\hline PEEK 150PF & $40 \pm 1$ \\
\hline PEEK 450PF & $46 \pm 1$ \\
\hline
\end{tabular}

Table 2. AOR results on the powder materials PEK HP3, thermally conditioned PEEK 150PF and 450PF

PEK HP3 is an optimised commercial powder material for HT-LS and therefore its AOR value was considered as the optimal number desired on potential new HT-LS grades. As it can be noticed from Table 2, the AOR test allowed the identification of the flow differences of the two thermally conditioned PEEK grades from the benchmark material PEK HP3. PEEK 150PF and PEK HP3 showed very similar performance, while PEEK 450PF showed a slightly higher $A O R$ value ( $4^{\circ}$ higher). These results can be explained by taking into consideration the combined effect of material properties and particle morphology of the powders under test. Material properties such as dielectric constant and refractive indices affect the extent of inter-particle forces, i.e. the higher the inter-particle forces, the less free-flowing behaviour of a powder. The flow performance is also influenced by the particle morphology of a given material, i.e. the more the shape descriptors of roundness, circularity, aspect ratio and solidity equate those of a spherical body, the higher the flowability will be. More details on this test and results are provided by the author in a previous work [5].

\subsection{Crystallinity content and crystal structure results}

\subsubsection{Powder and pellets analysis}

The melting and crystallisation peak temperatures, the crystallinity content evaluated by DSC analysis of the raw materials are listed in Table 3.

\begin{tabular}{|l|l|l|l|l|}
\hline Parameter & PEK HT22 & PEK HP3 & PEEK 150PF & PEEK 450PF \\
\hline Melting peak $\left({ }^{\circ} \mathrm{C}\right)$ & $371.0 \pm 0.4$ & $372.0 \pm 0.2$ & $343.6 \pm 6.4$ & $339.0 \pm 0.5$ \\
\hline Crystallisation peak $\left({ }^{\circ} \mathrm{C}\right)$ & $331.8 \pm 0.4$ & $332.2 \pm 0.3$ & $301.8 \pm 0.4$ & $295.6 \pm 0.6$ \\
\hline DSC crystallinity $(\%)$ & $49.6 \pm 1.8$ & $48.7 \pm 5.5$ & $46.7 \pm 5.2$ & $47.3 \pm 5.3$ \\
\hline
\end{tabular}

Table 3. Melting temperature, crystallisation temperature and crystallinity content of powder and pellet (PEK HT 22) samples

The PEK grades, PEK HT22 (pellets) and PEK HP3 (powder), show the highest melting and crystallisation peak temperatures as a consequence of the different chemical structure between PEK and PEEK [26]. In terms of crystallinity contents all the materials showed similar results.

According to the literature $[1,13,27]$ an ideal polymeric candidate for LS has a DSC thermal scan where melting and crystallisation regions do not overlap, thus identifying a temperature range where the polymer can easily processed. This phenomenon is known as "Super-cooling window" and it is shown in Figure 7. 


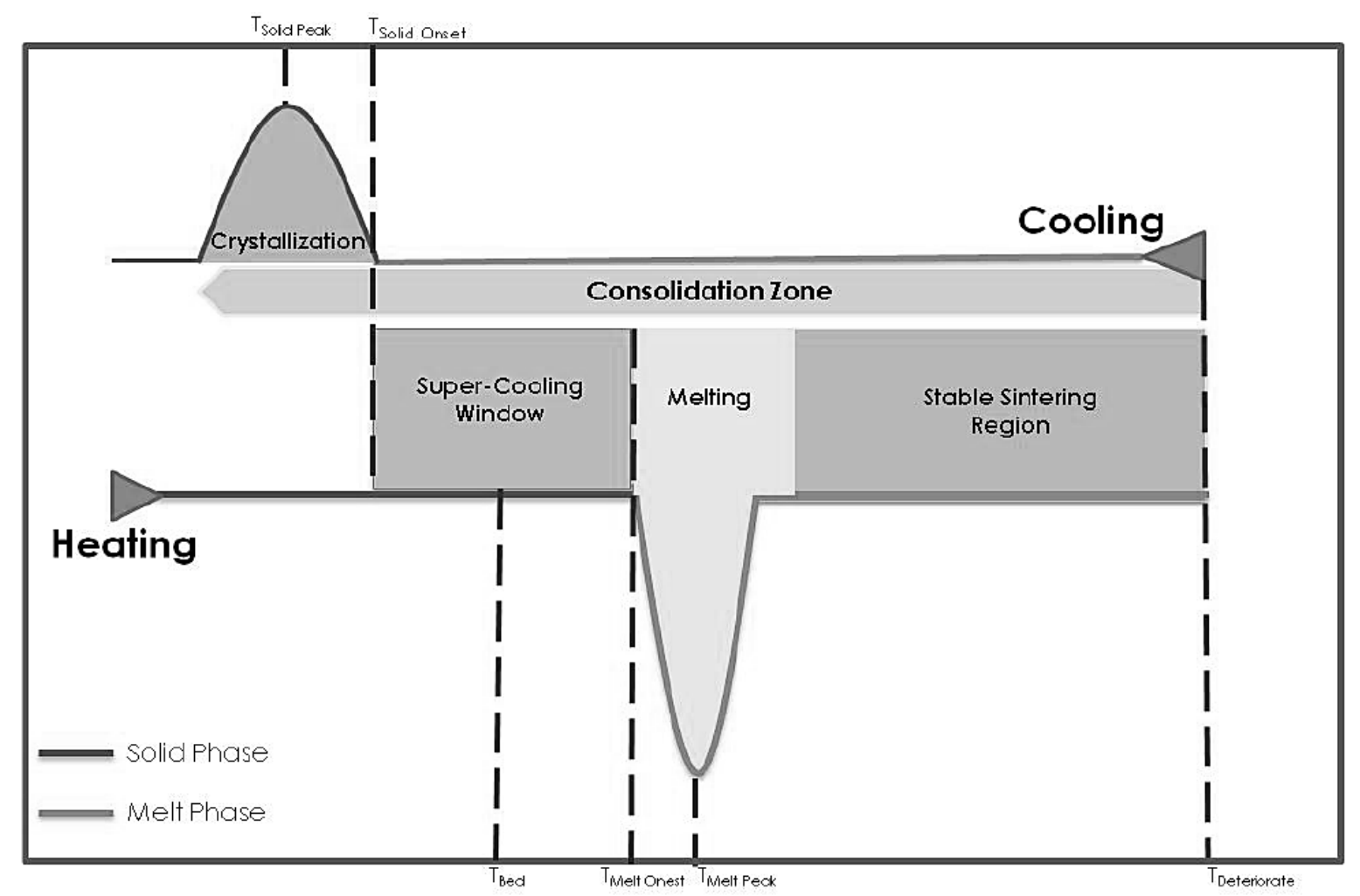

Figure 7. DSC thermal scan of an ideal polymer for LS processing. The super-cooling window, defined as the temperature interval between the onset melting temperature and the onset crystallisation temperature, is considered a key requirement for polymers in LS [25]

It is interesting to notice though that this requirement does not seem to be crucial for HT-LS processing with HTP. As shown in Figure 8, the commercial PEK HP3 grade does not show a super-cooling window and similar behavior is shown by PEEK 150PF and 450PF, shown in Figure 9. This is a surprising result but not unique. Vasquez et al. [25] also questioned the validity of the super cooling window when introducing amorphous polymers into LS. Amorphous polymers do not undergo through melting and crystallisation phenomena and therefore the super cooling window principle cannot be applied. However these polymers can still be successfully laser sintered. 


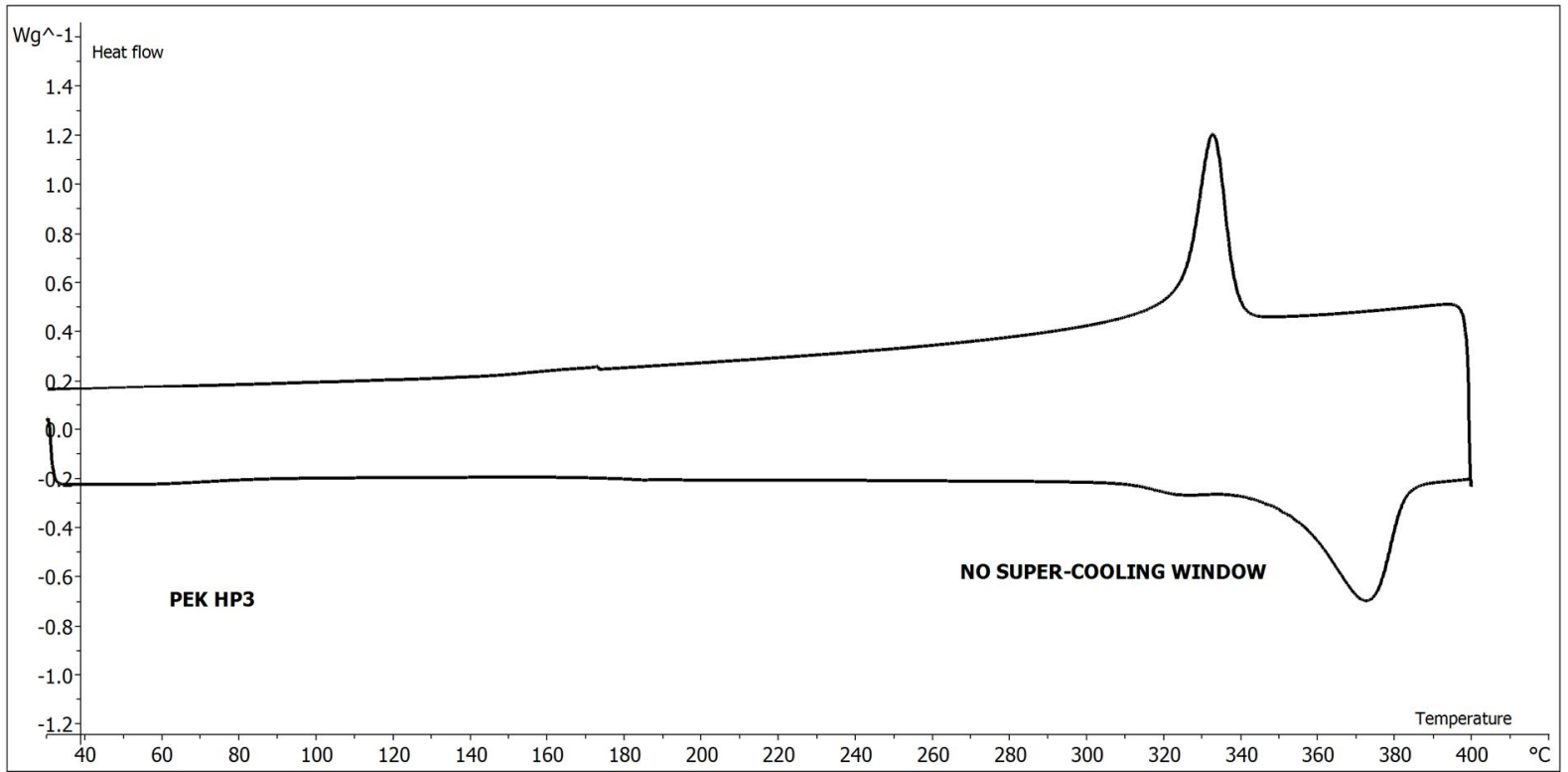

Figure 8. DSC thermal profile of PEK HP3 showing the lack of a super-cooling window

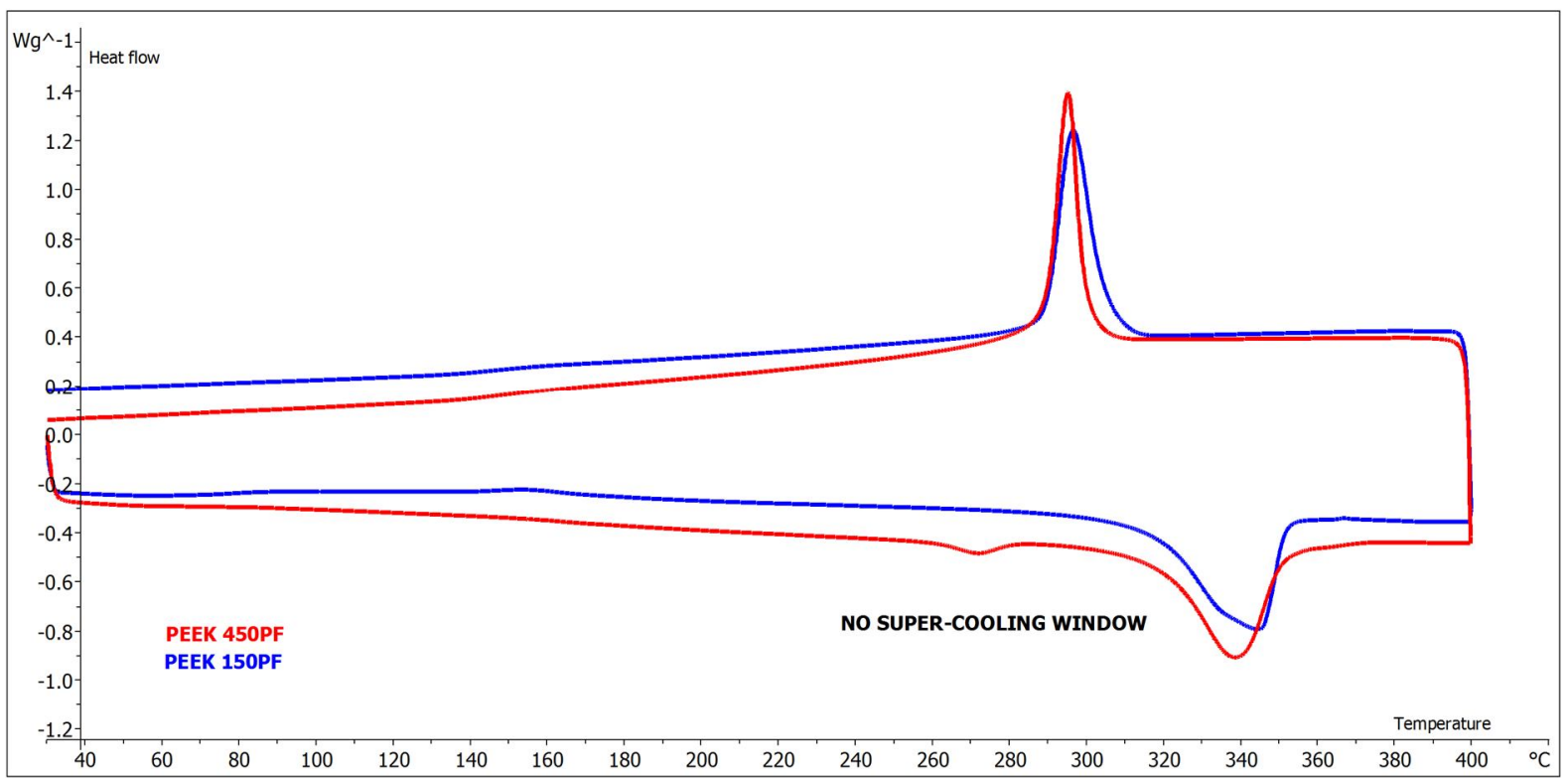

Figure 9. DSC thermal scans of PEEK 150PF and 450PF. Both materials do not exhibit a super-cooling window

\subsubsection{Crystalline morphology of the manufactured parts}

The crystallinity content, crystallisation peak temperature and crystal size of the manufactured samples, found using DSC and XRD analyses, are listed in Table 4. PEK samples manufactured by injection moulding are refereed as "IM -PEK", while PEK and PEEK samples manufactured by HT-LS are indicated with the prefix "HTLS".

\begin{tabular}{|c|c|c|c|c|}
\hline Parameter & IM-PEK & $\begin{array}{c}\text { HT-LS } \\
\text { PEK HP3 }\end{array}$ & $\begin{array}{c}\text { HT-LS } \\
\text { PEEK 150PF }\end{array}$ & $\begin{array}{c}\text { HT-LS } \\
\text { PEEK 450PF }\end{array}$ \\
\hline DSC crystallinity (\%) & $35.4 \pm 0.3$ & $58.0 \pm 3.2$ & $50.8 \pm 4.8$ & $49.5 \pm 5.0$ \\
\hline XRD crystallinity (\%) & $40.2 \pm 1.4$ & $53.3 \pm 1.0$ & $60.1 \pm 2.7$ & $62.7 \pm 3.2$ \\
\hline XRD crystal size (nm) & $9.2 \pm 2.7$ & $14.9 \pm 3.5$ & $17.1 \pm 3.8$ & $15.5 \pm 3.6$ \\
\hline
\end{tabular}

Table 4. Crystallinity and crystal size results of the manufactured samples 
The crystal size between IM-PEK and HT-LS PEK HP3 was found to be very different. HT-LS PEK samples showed bigger crystals than IM -PEK samples. This result could be explained by taking into consideration the difference in cooling profile between the two processes. A long and slow cooling phase is present in HT-LS allowing the crystals to grow more than in the IM process where instead, the material is rapidly cooled within the mould before being expelled. This is confirmed also by the lower crystallinity of IM samples in comparison with the HT-LS samples. It can be noticed that the crystallinity values obtained by XRD and DSC analyses are different. While in XRD analysis the crystallinity is measured under a constant temperature ("static measurement"), the sample crystallinity of a sample under DSC analysis is measured under a given thermal profile ("dynamic measurement"). In addition, XRD is a surface measurement where the DSC provides data from the bulk of the sample under test. According to Zimmerman and Konnecke [22], DSC analysis should be the best method for studying the crystallinity of PAEK polymers (PEEK, PEK, etc.).

\subsection{MFI results}

The M FI experimental results of PEEK grades and PEK HP3 are listed in Table 5.

\begin{tabular}{|c|c|c|}
\hline Material & Temperature $\left({ }^{\circ} \mathbf{C}\right)$ & Average M FI (g/ $\mathbf{1 0}$ min) \\
\hline PEK HP3 & 395 & $10.73 \pm 0.11$ \\
\hline PEEK 450PF & 365 & $1.83 \pm 0.04$ \\
\hline PEEK 150PF & 365 & $27.92 \pm 0.97$ \\
\hline \multicolumn{2}{|c|}{ Table 5. MFI results } \\
\hline
\end{tabular}

Table 5. M FI results

As expected from the material datasheets [28, 29], PEEK 150PF shows the highest value of M FI, while PEEK 450PF the lowest. PEEK 150PF is the least viscous polymers, 450PF the most viscous and PEK HP3 presents a value amongst the results of the two. A higher melt viscosity (low M FI value)is considered a consequence of a higher molecular weight [30]. In LS of semi-crystalline polymers, a low molecular weight has been reported to be preferable for good inter-particle bonding, but at the same time it is known that a high molecular weight leads to less shrinkage and subsequently higher dimensional accuracy [2]. Although it is known that high molecular weight is responsible for higher mechanical performance in polymers [31], the relationship between molecular weight, material viscosity and mechanical properties specifically in LS has not fully explored yet.

\subsection{Hot stage microscopy results}

The results of the first technique used in the hot stage microscopy testing for the materials PEK HP3, PEEK 150PF and PEEK 450PF are shown in Table 6.

\begin{tabular}{|c|c|c|}
\hline Material & Coalescence starting temperature $\left({ }^{\circ} \mathbf{C}\right)$ & Recording starting temperature $\left({ }^{\circ} \mathbf{C}\right)$ \\
\hline PEK HP3 & 380 & 360 \\
\hline PEEK 450PF & 340 & 320 \\
\hline PEEK 150PF & 340 & 320 \\
\hline
\end{tabular}


Table 6. Particle coalescence: results of the first method aiming to identify coalescence and recording start temperature in the hot stage device

This technique aimed to identify the onset coalescence temperature in the hot stage microscope under a

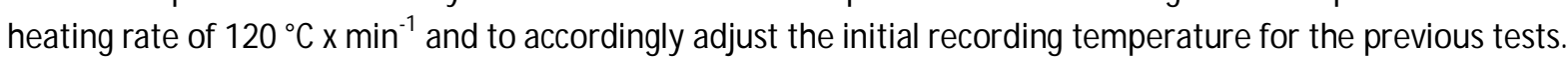
PEK HP3 showed a coalescence onset temperature of $380^{\circ} \mathrm{C}$, while both PEEK grades started to exhibit the formation of an inter-particle neck at $340^{\circ} \mathrm{C}$. The starting temperatures were therefore set at $360{ }^{\circ} \mathrm{C}$ for PEK and at $320^{\circ} \mathrm{C}$ for both PEEK grades.

The second approach used for studying particle coalescence aimed to understand the coalescence velocity or coalescence rate of PEK HP3, PEEK 150PF and 450PF for a two particle set-up. Measurements of the initial diameters of the particles and continuous measurements of the neck bonding were performed during each experiment. The maximum diameter of a particle was chosen as the initial diameter, as there is not a correct or preferred way of choosing a particle diameter. The ratio between neck diameter or neck length $(x)$ and the average (D) of the particle diameter of the two particles (D1 and D2) under test was evaluated in each test as shown in specific previous literature [32]. An example of particle diameters and neck length are shown in Figure 10. 

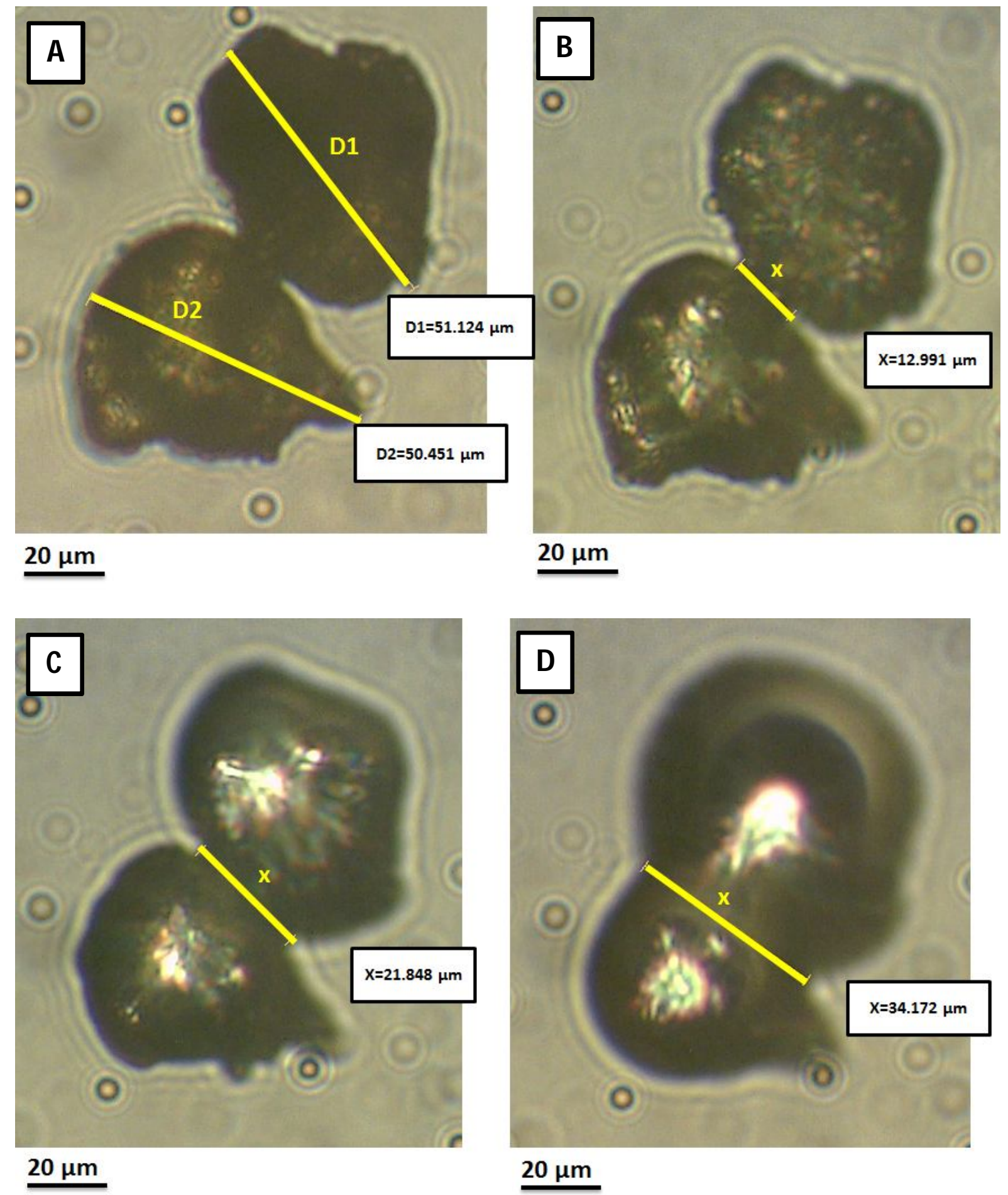


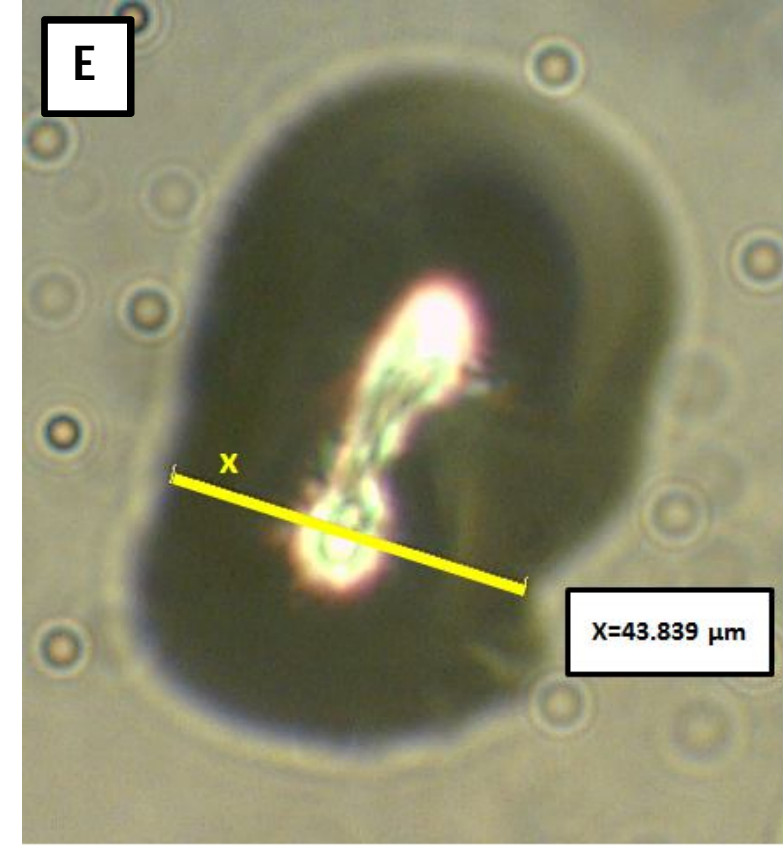

$20 \mu \mathrm{m}$

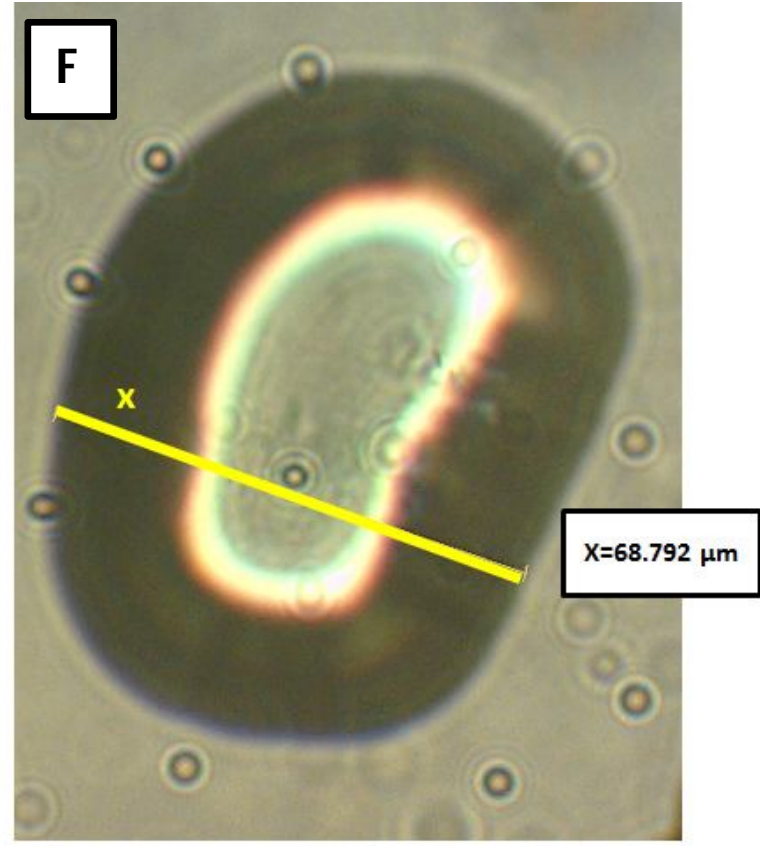

$20 \mu \mathrm{m}$

Figure 10. Particle coalescence of PEK HP3 particles. Measurements of the neck formation during coalescence: D1 and D2 particle diameters, $X$ neck length, $D=(D 1+D 2) / 2$. A-Room temperature, $B$ to $F$ during coalescence in the temperature range $380-400{ }^{\circ} \mathrm{C}$

A minimum number of four tests was performed on each material allowing to identify the significant differences between the materials. As the repeats were found to be consistent only one curve is displayed for each material in Figure 11 and Figure 12.

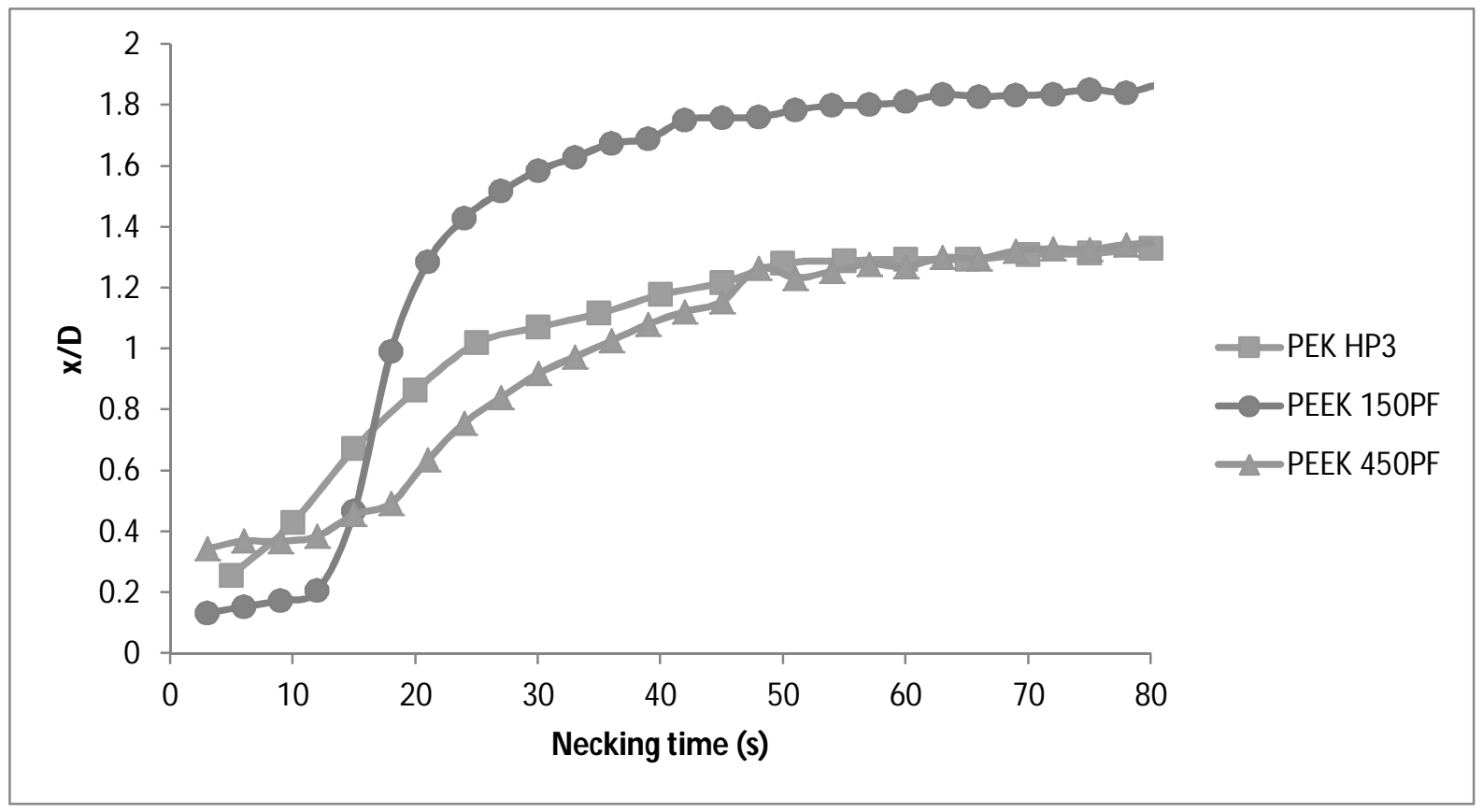

Figure 11. Coalescence results of PEK HP3, PEEK 150PF and PEEK 450PF particles. 


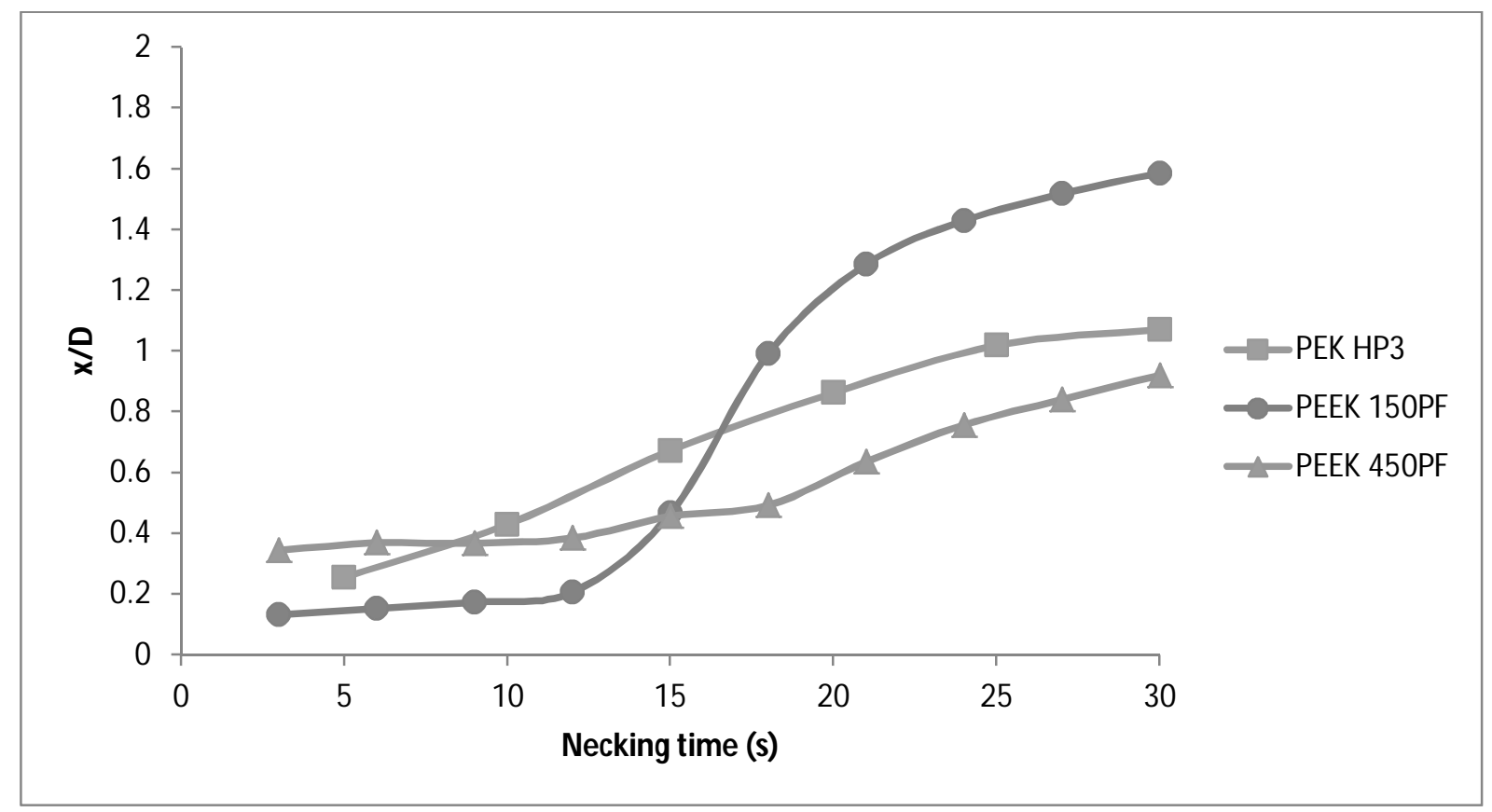

Figure 12. First 30s of the coalescence process of PEK HP3, PEEK 150PF and 450PF particles

The ratio between the neck formation and the average of the particles diameters of the two particles under test is plotted against the neck formation time. The " $0 \mathrm{~s}$ " time corresponded to the formation of a neck between the two particles, while the end of the " $80 \mathrm{~s}$ " time in Figure 11 refers to the end of particle coalescence. The end could either correspond to a time when the final particle stopped growing in diameter or to when the final particles reached other adjacent new particles and fused together as a uniform film.

As it can be seen in Figure 11 and Figure 12, the slope of three curves is significantly different.

The first 15 seconds of the coalescence process show a very different behaviour between the two PEEK grades and PEK HP3. Although the neck formation is in progress between the PEEK 150PF and 450PF particles, the growth is very slow, if any. This could be due to the changes in the particle morphology such as roughness, porosity and overall shape, which dominate this part of the process. As shown in Figure 2, PEK HP3 particles have a much smoother surface and better overall shape descriptors (circularity, aspect ratio, etc.) therefore encountered fewer changes at the beginning of the coalescence process.

PEEK 15OPF showed highest slope and therefore the highest necking formation rate, followed by PEK HP3 and PEEK 45OPF over the same time interval. This result suggests that during a HT-LS building operation particles of PEEK 15OPF will melt and coalescence faster than the established PEK HP3 polymer. This behaviour is believed to be a consequence of the considerable lower viscosity of PEEK 150PF than that of PEK HP3 (Table 5) and should be considered carefully in relation with the duration of the laser exposure and post sintering phase during HT-LS processing.

PEEK 450PF and PEK HP3 end with a similar neck growth, however the initial part of the neck formation is very different. Similar to PEEK 150PF, there is little coalescence takin place in the first 15s in PEEK 450PF, followed by an increase in the neck growth at a slightly lower rate than PEK HP3. In addition to the particle morphology (as described above), it is believed that the high viscosity of the polymer contributes to the overall slower neck growth. 
It is interesting to notice also that the neck growth/diameter ratios for the three materials are significantly different: PEEK 150PF reaches an approximately 1.8 ratio while both PEEK 450PF and PEK HP3 settle at approximately 1.2. It is important to mention that the particle diameter used for the calculations was the one measured at the beginning of the experiments when the particles were first placed on the hot stage. It is difficult to draw conclusions on the neck/diameter ratio differences, but it is evident that the particle morphology at the beginning and throughout the coalescence process is very important can influence the performance of the laser sintered parts.

On the basis of these results, it also appears that PEEK 150PF might not need a post sintering phase and therefore a shorter layer time than PEK HP3 during HT-LS processing, while PEEK 450PF might require a similar if not longer post-sintering phase than the established PEK HP3 and a longer layer time.

\subsection{Tensile test results}

The tensile strength results of IM- PEK, HT-LS PEK HP3, HT-LS PEEK 15OPF and HT-LS PEEK 450PF are listed in Table 7.

\begin{tabular}{|c|c|}
\hline Material & Tensile strength (M Pa) \\
\hline IM -PEK & $98 \pm 1$ \\
\hline HT-LS PEK HP3 & $88 \pm 7$ \\
\hline HT-LS PEEK 150PF & $41 \pm 5$ \\
\hline HT-LS PEEK 450PF & $63 \pm 5$ \\
\hline
\end{tabular}

Table 7. Tensile strength results

IM -PEK was used as benchmark material for the performances of the laser sintered components. HT laser sintered PAEK samples showed a drop of tensile strength when compared to IM -PEK, indicating the manufacturing process as reason for this observed behaviour. In IM, a fully molten polymer is injected under pressure into a cold mould. In LS and HT-LS the raw material lies in a powder bed and a laser consolidates specific areas across every layer, but there is no pressure that applies further compaction. It is well known that highly dense parts lead to higher mechanical properties than parts made of the same material but showing the presence of some porosity in the microstructure. The difference therefore in the two manufacturing processes is likely to be the reason why IM specimens showed higher tensile strength.

The highest values of tensile strength in HT laser sintered specimens was exhibited by PEK HP3, followed by PEEK 45OPF and PEEK 150PF. The difference in the chemical composition between PEK HP3 and the two grades of PEEK contributes towards the variation in strength [21]. HT laser sintered PEEK 450PF specimens present significant higher tensile strength than $\mathrm{HT}$ laser sintered PEEK 150PF. As the only difference between the two materials was the material viscosity (same polymer-Table 1, same crystallinity-Table 5), it is reasonable to consider the latter responsible for the different tensile behaviour. The fact that the crystalline morphology analysis reveals similar structures is an indication that the molecular weight does not play a significant effect here and therefore this is the case where the mechanical behavior is primarily related with the particle morphology at the beginning and throughout the LS process as well as the polymer viscosity.

Shi et al. [10] suggested that the lower molecular weight of the two grades of PS led to denser LS components of higher quality, contradicting somehow what was found experimentally in this work. However, they did not investigate in detail the tensile properties of their PS laser sintered samples. 
Interestingly, it can also be noted that the standard deviation of the tensile strength results is larger in the HT laser sintered units. This fact could be due to the variation in powder morphology and control of the thermal environment. Particle morphology is known to affect the flow behaviour and layer spreading results in LS [5, 6]. Variations in the particle morphology can lead to high and low density areas across the layer of powder during a LS manufacturing operation. This fact in turn can cause some specimens to be denser and perform better in tensile strength or less dense with weaker microstructures exhibiting porosity and consequential lower tensile strength.

\subsection{DMA results}

Figure 13 and Figure 14 show the storage modulus, the loss modulus and the tan $\delta$ of IM -PEK and HT-LS PEK HP3.

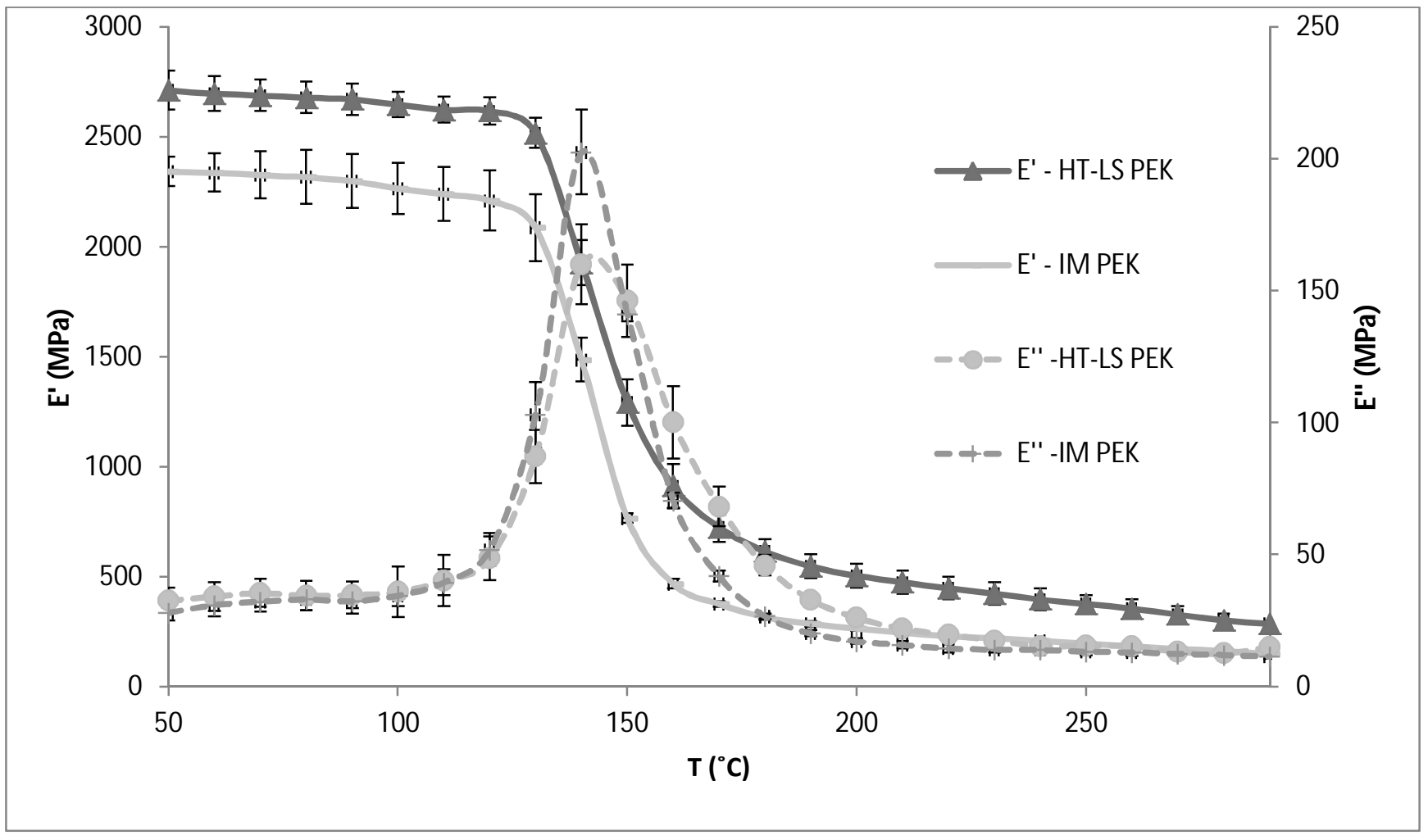

Figure 13. Storage modulus (E') and Loss modulus (E') of PEK samples produced by HT-LS (HT-LS PEK) and by IM (IM PEK)

Below the glass transition temperature $\left(143^{\circ} \mathrm{C}\right)$, the storage modulus of HT-LS PEK HP3 is approximately $10 \%$ higher than IM-PEK as a result of increased crystallinity of HT-LS manufacturing (Figure 13). IM-PEK and LS PEK HP3 samples showed similar tan $\delta$ for temperatures below $120^{\circ} \mathrm{C}$ (Figure 14). After this temperature, injection moulded samples exhibited a greater damping effect than the HT laser sintered specimens. This could be explained by the higher crystallinity content and crystal size recorded in the HT laser sintered samples, implying that the volume of amorphous region is lower which in turn indicates reduced mobility of the molecular chains of the polymer. This property is translated in a lower damping event in the DM A profiles. In contrast, IM -PEK samples exhibited lower crystallinity and smaller crystals allowing a more plastic behaviour of the polymer overall, resulting in a much more pronounced damping peak. A similar effect on the damping peak 
was found by Zhang et al. [33] when studying and comparing the damping characteristics of laser sintered and compression moulded PA based composites.

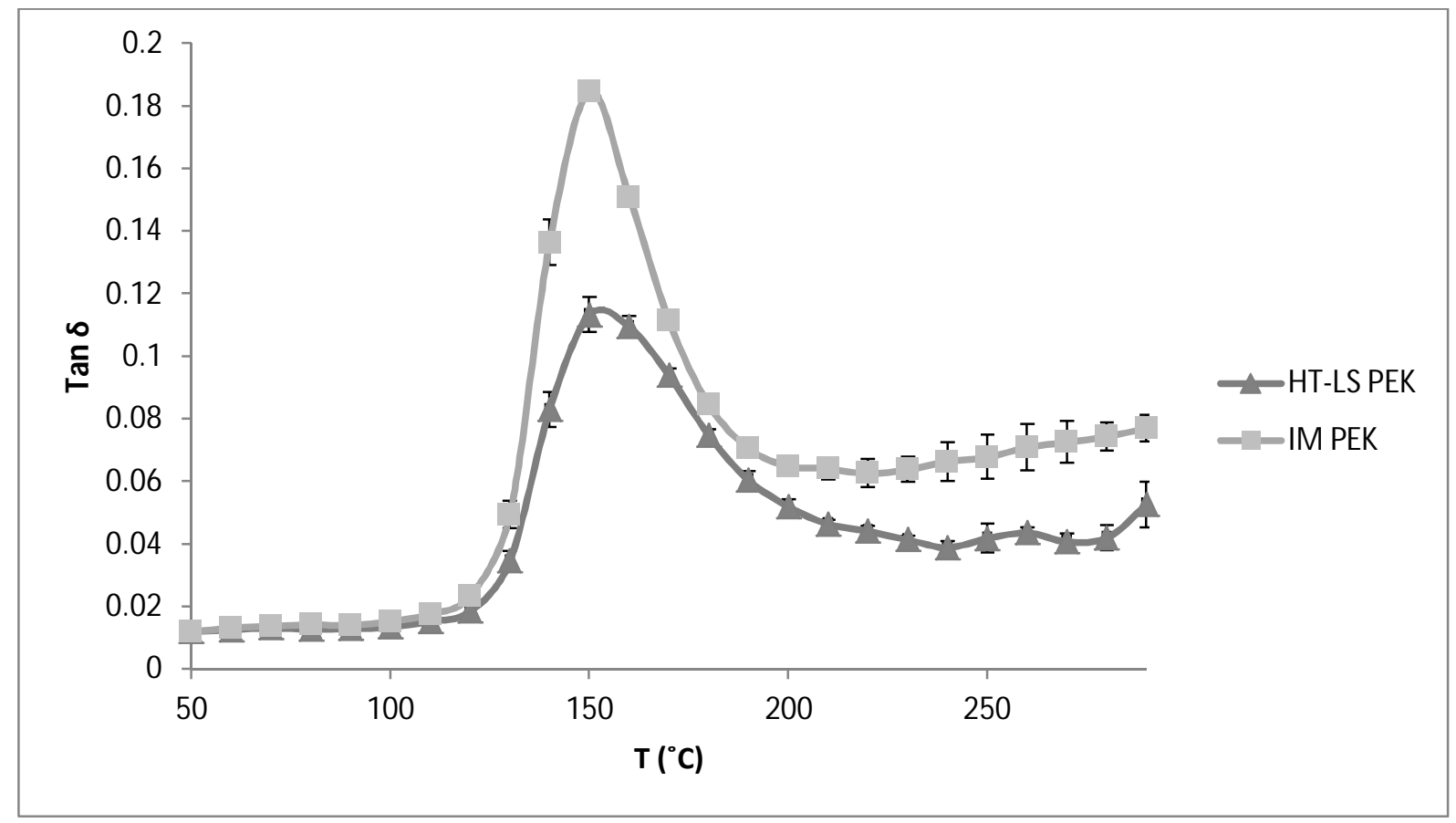

Figure 14. Damping factor (Tan $\delta$ ) of PEK samples produced by HT-LS (HT-LS PEK) and by IM (IM PEK)

The DM A behaviour of HT laser sintered PEK HP3, PEEK 150PF and 450PF specimens is shown in Figure 15 and Figure 16. 


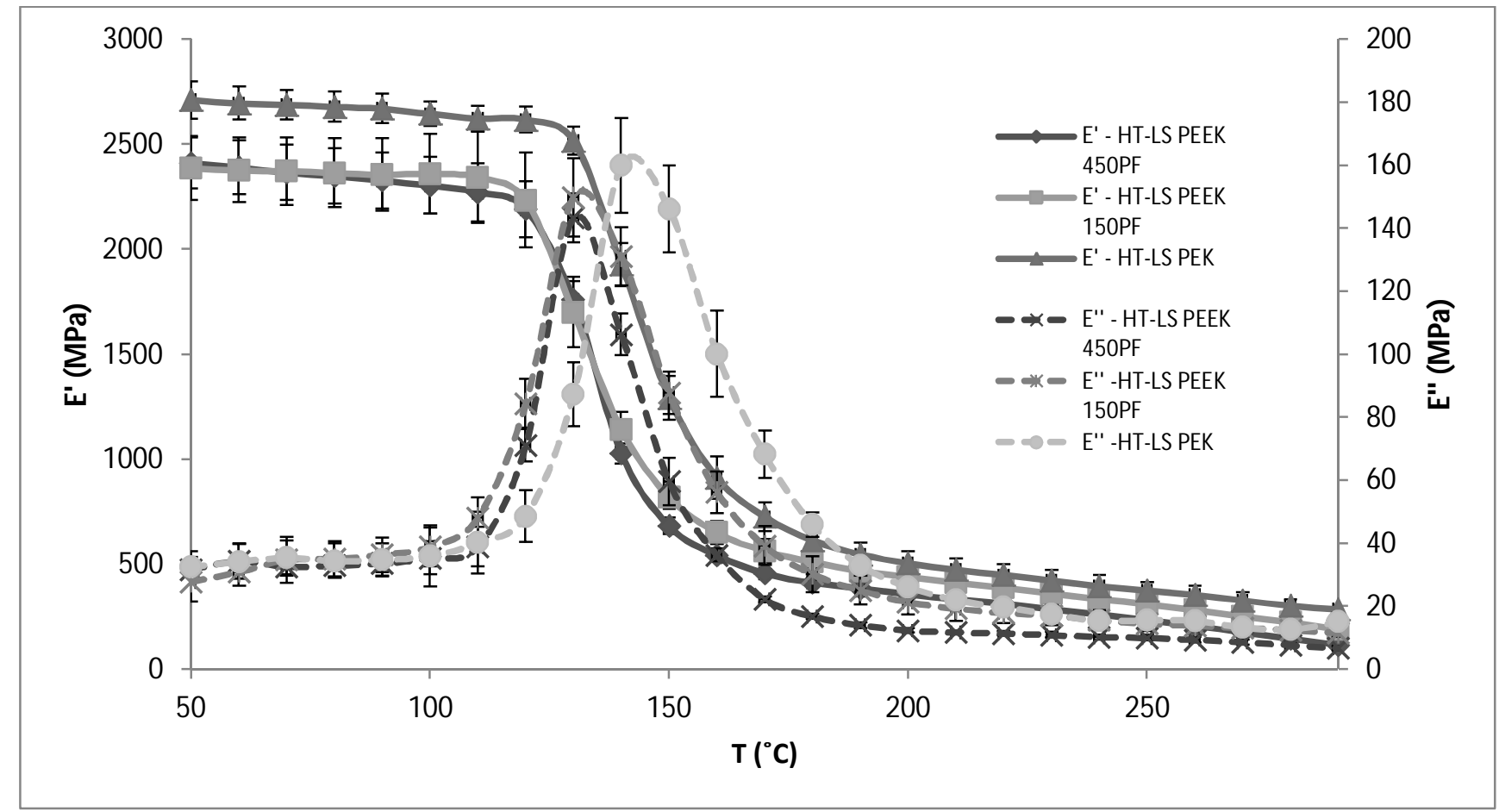

Figure 15. Storage modulus (E') and Loss modulus (E') of PEK, PEEK 150PF and PEEK 450PF samples made by HT-LS

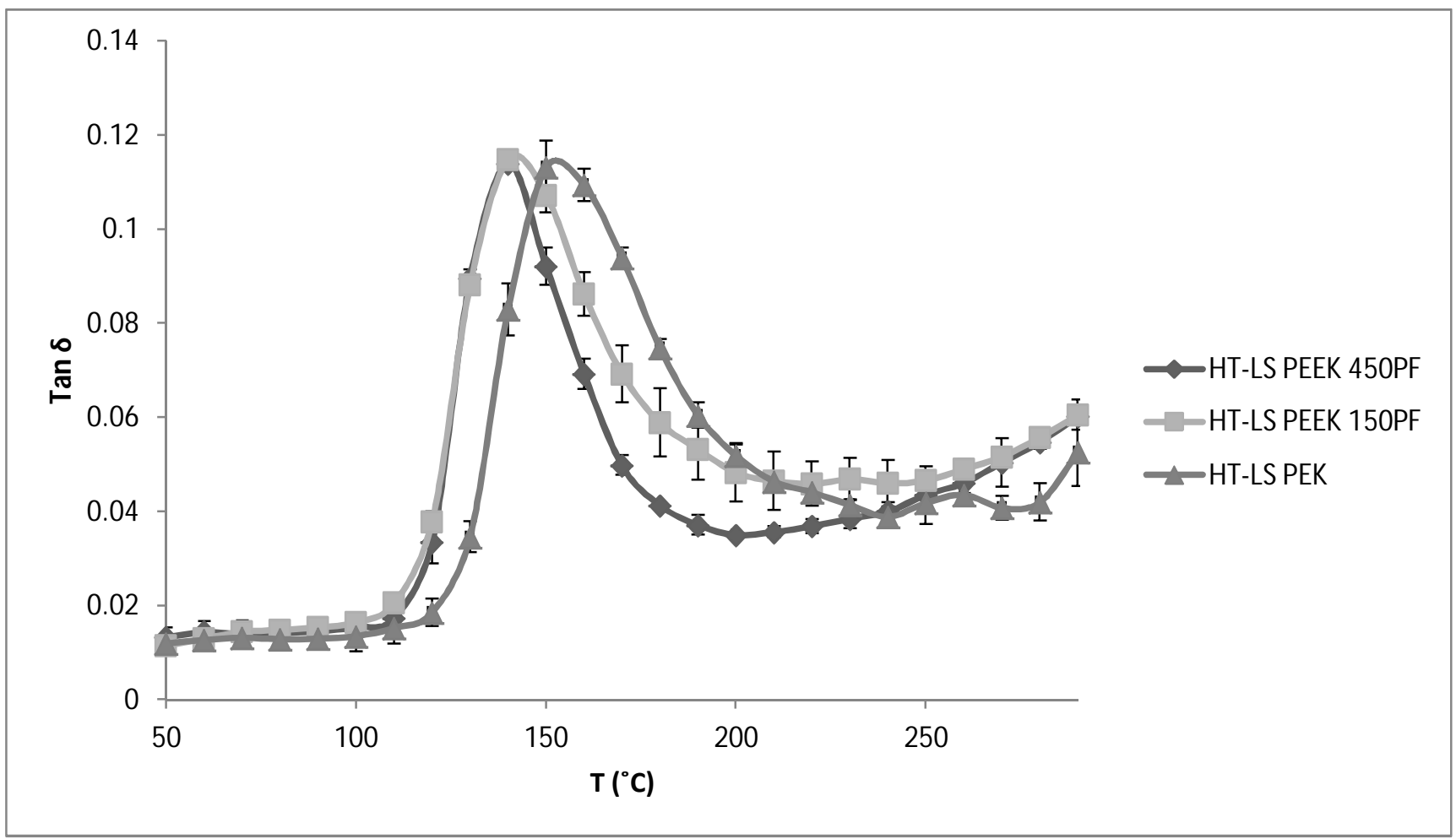

Figure 16. Damping factor (Tan $\delta$ ) of PEK, PEEK 150PF and PEEK 450PF samples made by HT-LS 
The differences in the chemical structure and crystallinity (due to the molecular weights) might be the cause for the slightly higher storage modulus in PEK HP3 than PEEK. The PEEK 150PF and 450PF had similar storage modulus values. Also, as PEK has a higher glass transition temperature than PEEK $\left(164{ }^{\circ} \mathrm{C}\right.$ and $143^{\circ} \mathrm{C}[28,29$, 34], respectively), storage and loss moduli were shifted towards higher temperature values .

\section{Conclusion}

HT-LS is a manufacturing process whose potential and applications can be stalled if new materials are not developed. This work provides new insights into the role of microstructure, molecular weight and melt viscosity of polymeric powdered materials in HT-LS manufacturing by comparison with IM parts properties. The key outcome of this paper is that the melt viscosity of materials has been quantitatively proven to affect the rate of sintering such as the inter-particle neck formation, indicating for less viscous polymers a faster coalescence. In the same analysis, it was noticed that the particle morphology affect the initial phase of the coalescence process. The materials with higher viscosity (PEK HP3 and PEEK 450PF) showed higher tensile strength than the grade with the lowest viscosity when processed in HT-LS. The combined results of tensile strength, viscosity measured through $\mathrm{M} \mathrm{Fl}$ and neck ratio formation are shown in Figure 17. M oving away from the current material properties, it is envisaged that an ideal material should combine low viscosity (high M FI) as exhibited by the PEEK 150PF for good particle coalescence (and in general good polymer processing), good particle morphology and high mechanical performance as presented by PEK HP3. Developments in the chemistry of PAEK polymers such as those described by M anolakis et al. [35] could lead to materials that will be easier to process and with enhanced mechanical performances. 


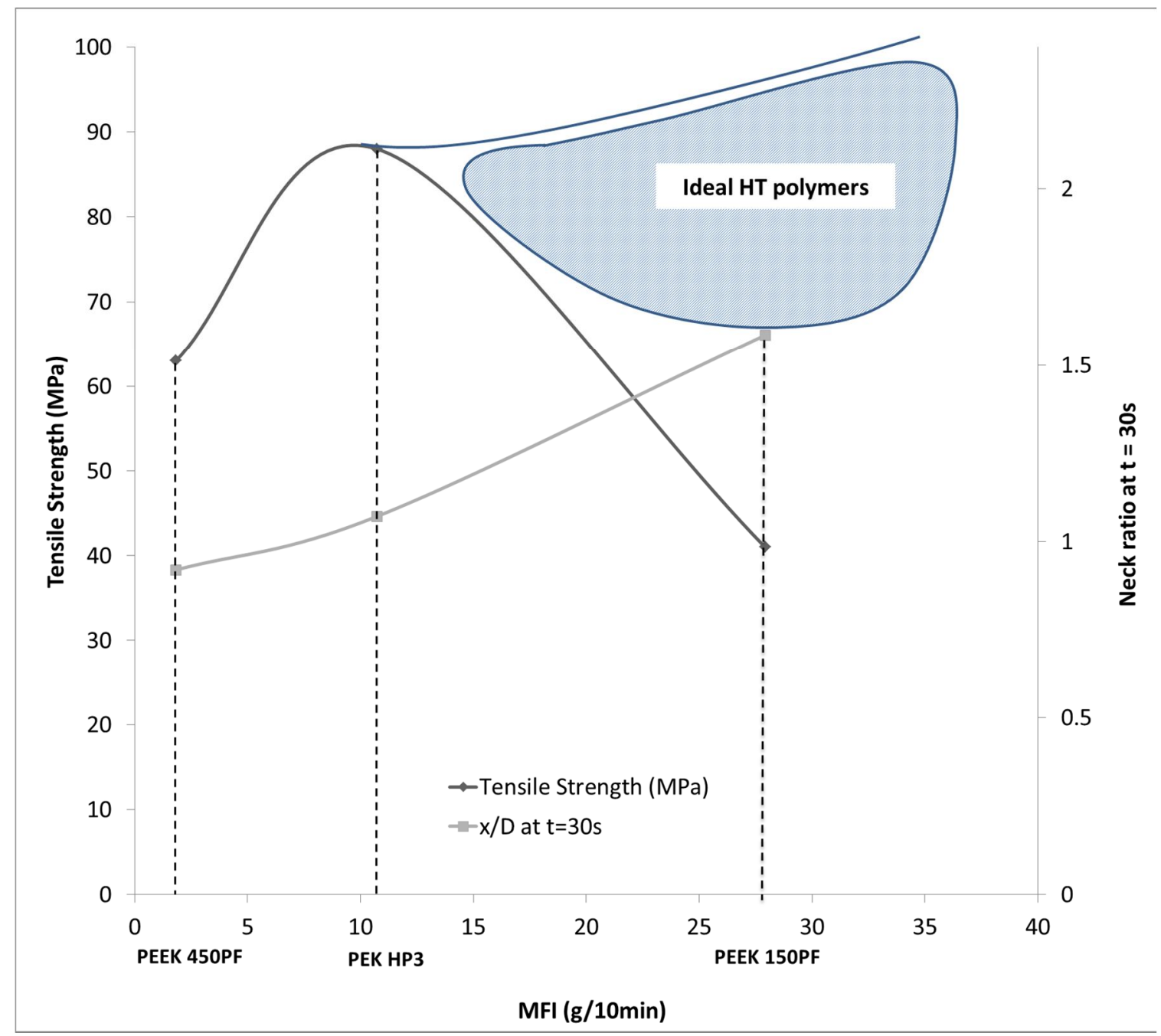

Figure 17. Plot showing the relationship found between tensile performance, particle sintering (neck ratio x/D) and viscosity (M Fl)

\section{Conflict of interest}

The authors declare that they have no conflict of interest.

\section{References}

1. Goodridge, R.D., C.J. Tuck, and R.J.M. Hague, Laser sintering of polyamides and other polymers. Progress in M aterials Science, 2012. 57(2): p. 229-267.

2. Kruth, J.P., et al., Consolidation phenomena in laser and powder-bed based layered manufacturing. CIRP Annals - M anufacturing Technology, 2007. 56(2): p. 730-759.

3. Beard, M., et al. Material characterisation of Additive M anufacturing components made from a polyetherketone (PEK) high temperature thermoplastic polymer. in Advanced Research in Virtual and Rapid Prototyping (VRAP). 2011. Leiria, Portugal: CRC Press.

4. Berretta, S., K.E. Evans, and O. Ghita, Processability of PEEK, a new polymer for High Temperature Laser Sintering (HT-LS). European Polymer Journal, 2015. 68: p. 243-266.

5. Berretta, S., O. Ghita, and K.E. Evans, M orphology of polymeric pow ders in Laser Sintering (LS): From Polyamide to new PEEK powders. European Polymer J ournal, 2014. 59: p. 218-229. 
6. Berretta S, G.O., Evans K.E., Anderson A. and Newman C., Size, shape and flow of powders for use in Selective Laser Sintering (SLS) in Advanced research in virtual and rapid prototyping, P.J. Bártolo, Editor 2013, CRC Press: Leiria, Portugal.

7. Ghita, O., James, E., Davies, R., Berretta, S., Singh, B., Flint, S. and Evans, K. E., High Temperature Laser Sintering (HT-LS): An investigation into mechanical properties and shrinkage characteristics of Poly (Ether Ketone) (PEK) structures. M aterials \& Design, 2014. 61: p. 124-132.

8. Ghita, O.R., et al., Physico-chemical behaviour of Poly (Ether Ketone) (PEK) in High Temperature Laser Sintering (HT-LS). Journal of M aterials Processing Technology, 2013.

9. Wang, Y., E. James, and O. Ghita, Glass Bead Filled Polyetherketone (PEK) composite by High Temperature Laser Sintering (HT-LS). M aterials \& Design, 2015: p. pp. 545-551.

10. Shi, Y., et al., Effect of the properties of the polymer materials on the quality of selective laser sintering parts. Proceedings of the Institution of M echanical Engineers, Part L: Journal of Materials Design and Applications, 2004. 218(3): p. 247-252.

11. M isra, G.S., Introductory Polymer Chemistry1993: J. Wiley \& Sons.

12. Yan, C., et al., Preparation, characterisation and processing of carbon fibre/ polyamide-12 composites for selective laser sintering. Composites Science and Technology, 2011. 71(16): p. 1834-1841.

13. Wendel, B., et al., Additive Processing of Polymers. M acromolecular Materials and Engineering, 2008. 293(10): p. 799-809.

14. Rietzel D., W.A., Dietmar Drummer, Tim A. Osswald, Polymer powders for Selective Laser Sintering - Production and Characterization.

15. Ku CW, G.I.a.C.W., Selective laser sintered castform polystyrene with controlled porosity and its infiltration characteristics by red wax, in 13th solid freeform fabrication (SFF) symposium, 5-7 August 20022002: University of Texas at Austin, Texas. p. pp. 107-114.

16. Materialise. http://www.materialise.com/. [cited 2016 January].

17. 3DSystems. http://www.3dsystems.com/. [cited 2015 April].

18. EOS. http://www.eos.info/en/home.html. [cited 2015 April].

19. Victrex. http://www.victrex.com. [cited 2015 April].

20. Leuterer M., M.F., Pfister A., PAEK pow der, in particular for the use in a method for a layerwise manufacturing of a three-dimensional object, as well as method for producing it, 2012.

21. Kurtz, S.M . and J.N. Devine, PEEK biomaterials in trauma, orthopedic, and spinal implants. Biomaterials, 2007. 28(32): p. 4845-4869.

22. Zimmermann, H.J. and K. Könnecke, Crystallization of poly(aryl ether ketones): 3 . The crystal structure of poly(ether ether ketone ketone) (PEEKK). Polymer, 1991. 32(17): p. 3162-3169.

23. Mackenzie, J.K. and R. Shuttleworth, A Phenomenological Theory of Sintering. Proceedings of the Physical Society. Section B, 1949. 62(12): p. 833.

24. Bellehumeur, C.T., M.K. Bisaria, and J. Vlachopoulos, An experimental study and model assessment of polymer sintering. Polymer Engineering \& Science, 1996. 36(17): p. 21982207.

25. Vasquez, M., Analysis and development of new materials for polymer laser sintering, 2012, Loughborough University.

26. Harsha, A.P. and U.S. Tewari, Tribo performance of polyaryletherketone composites. Polymer Testing, 2002. 21(6): p. 697-709.

27. Gibson, I. and D.P. Shi, M aterial properties and fabrication parameters in selective laser sintering process. Rapid prototyping journal, 1997. 3(4): p. 129-136.

28. Victrex, PEEK 150 PF datasheet.

29. Victrex, PEEK 450 PF datasheet.

30. Rosato, D.V. and M.G. Rosato, Injection Molding Handbook2000: Kluwer Academic Publishers. 
31. Mitchell, B.S., An Introduction to Materials Engineering and Science for Chemical and Materials Engineers2004: Wiley.

32. Vasquez M., H.N., Haworth B., Laser Sintering Processes: Practical Verification of Particle Coalescence for Polyamides and Thermoplastic Elastomers. ANTEC, 2010.

33. Zhang, Y., Hao, L., Savalani, M. M., Harris, R. A. and Tanner, K. E., Characterization and dynamic mechanical analysis of selective laser sintered hydroxyapatite-filled polymeric composites. Journal of Biomedical Materials Research Part A, 2008. 86A(3): p. 607-616.

34. EOS, EOS PEEK HP3 datasheet.

35. Manolakis, I., et al., Ring-opening polymerization in molten PEEK: transient reduction of meltviscosity by macrocyclic aromatic thioetherketones. Journal of Materials Chemistry, 2012. 22(38): p. 20458-20464. 